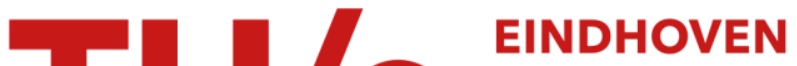 UNIVERSITY OF TECHNOLOGY
}

\section{The time-dependent capacitated profitable tour problem with time windows and precedence constraints}

\section{Citation for published version (APA):}

Sun, P., Veelenturf, L. P., Dabia, S., \& van Woensel, T. (2018). The time-dependent capacitated profitable tour problem with time windows and precedence constraints. European Journal of Operational Research, 264(3), 1058-1073. https://doi.org/10.1016/j.ejor.2017.07.004

DOI:

10.1016/j.ejor.2017.07.004

Document status and date:

Published: 01/02/2018

\section{Document Version:}

Accepted manuscript including changes made at the peer-review stage

\section{Please check the document version of this publication:}

- A submitted manuscript is the version of the article upon submission and before peer-review. There can be important differences between the submitted version and the official published version of record. People interested in the research are advised to contact the author for the final version of the publication, or visit the $\mathrm{DOI}$ to the publisher's website.

- The final author version and the galley proof are versions of the publication after peer review.

- The final published version features the final layout of the paper including the volume, issue and page numbers.

Link to publication

\section{General rights}

Copyright and moral rights for the publications made accessible in the public portal are retained by the authors and/or other copyright owners and it is a condition of accessing publications that users recognise and abide by the legal requirements associated with these rights.

- Users may download and print one copy of any publication from the public portal for the purpose of private study or research.

- You may not further distribute the material or use it for any profit-making activity or commercial gain

- You may freely distribute the URL identifying the publication in the public portal.

If the publication is distributed under the terms of Article 25fa of the Dutch Copyright Act, indicated by the "Taverne" license above, please follow below link for the End User Agreement:

www.tue.nl/taverne

Take down policy

If you believe that this document breaches copyright please contact us at:

openaccess@tue.nl

providing details and we will investigate your claim. 


\title{
The time-dependent capacitated profitable tour problem with time windows and precedence constraints
}

\author{
Peng Sun ${ }^{\mathrm{a}, *}$, Lucas P. Veelenturf ${ }^{\mathrm{a}}$, Said Dabia ${ }^{\mathrm{b}}$, Tom Van Woensel $^{\mathrm{a}}$ \\ ${ }^{a}$ School of Industrial Engineering, Operations, Planning, Accounting and Control (OPAC), Eindhoven University of Technology, Eindhoven 5600 MB, The \\ Netherlands \\ ${ }^{\mathrm{b}}$ Department of Information, Logistics and Innovation, Vrije Universiteit Amsterdam, Amsterdam 1081 HV, The Netherlands
}

\section{A R T I C L E I N F O}

\section{Article history:}

Received 16 August 2016

Accepted 3 July 2017

Available online $\mathrm{xxx}$

\section{Keywords:}

Transportation

Profitable tour problem

Pickup and delivery problem

Tailored labeling algorithm

Time-dependent travel times

\begin{abstract}
A B S T R A C T
We introduce the time-dependent capacitated profitable tour problem with time windows and precedence constraints. This problem concerns determining a tour and its departure time at the depot that maximizes the collected profit minus the total travel cost (measured by total travel time). To deal with road congestion, travel times are considered to be time-dependent. We develop a tailored labeling algorithm to find the optimal tour. Furthermore, we introduce dominance criteria to discard unpromising labels. Our computational results demonstrate that the algorithm is capable of solving instances with up to 150 locations ( 75 pickup and delivery requests) to optimality. Additionally, we present a restricted dynamic programing heuristic to improve the computation time. This heuristic does not guarantee optimality, but is able to find the optimal solution for 32 instances out of the 34 instances.
\end{abstract}

(c) 2017 Elsevier B.V. All rights reserved.

\section{Introduction}

The effective usage of empty vehicles' space is an important opportunity to increase the efficiency of urban transportation systems and to reduce traffic congestion, fuel consumption, and pollution. Companies (e.g., Uber) can generate extra income by renting out vehicles's empty space rising in their transportation processes. Several mobile applications are developed to improve the last-mile deliveries by involving the city's residents. For instance, Roadie created an on-the-way delivery network which is an online market where people post their required shipments and where anyone can offer to execute the shipment. DHL launched a platform called MyWays, enabling individuals to deliver packages with products ordered online directly to other end consumers. For those individuals with limited transportation resources, it is important to know which parcels are profitable to collect and deliver. On the one hand, serving a request may be attractive because it generates revenue. On the other hand, there is additional cost for serving the request. Consequently, it might not always be economically beneficial to serve a request. Moreover, customers are not always available to receive the ordered goods (i.e., shops are not open $24 / 7$ or e-commerce customers are not always home). Therefore, these customers propose specific time windows in which they

\footnotetext{
* Corresponding author.

E-mail addresses: P.Sun@tue.nl (P. Sun), L.p.veelenturf@tue.nl (L.P. Veelenturf) S.dabia@vu.nl (S. Dabia), T.v.Woensel@tue.nl (T. Van Woensel).
}

want to be served. These extra constraints make it a challenge for the independent courier to be everywhere on time. He has to select a subset of the requests to be served within the requested time windows. Furthermore, due to the limited capacity of the road network and increased traffic congestion, the travel speed is serious affected by these traffic fluctuations. This results in great variations in travel times and thereby on the arrival times at customers. Therefore, the travel time between locations is dependent on the time the driver departs (e.g., in rush hours traveling takes more time). Not considering the time-dependent travel time results in too late arrivals at the customers (see e.g., Taş, Dellaert, van Woensel, \& de Kok, 2014). All these complex situations illustrate the need for the independent couriers to have a tool to help them out in making decisions on which request to serve and which time to depart. However, scarce literature can be found that focus on this area.

This paper aims at building such a tool by modeling and solving a time-dependent capacitated profitable tour problem with time windows and precedence constraints. We consider a single vehicle with capacity limit and a set of requests which have a pick-up and a delivery node. Each pickup node and delivery node has its own time window in which it should be served. Moreover, a delivery node of a request can only be served after its pickup node is visited. For each served request a profit is collected. To capture travel speed variation during a day, a time-dependent travel time function is assigned to each edge linking two nodes. The objective is to determine the vehicle's tour starting and ending at the depot, and 


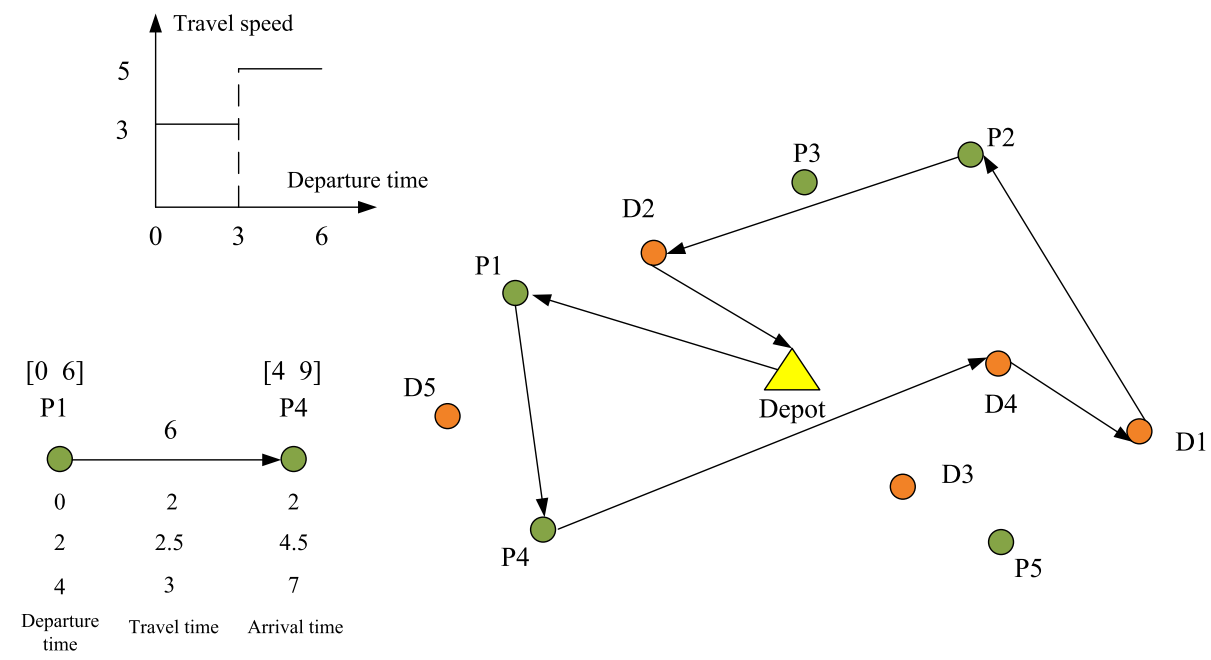

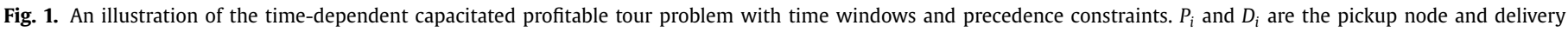
node of request $i$, respectively.

maximizing the difference between the total collected profits and total travel cost (see Fig. 1). Following the literature in this area, the tour's total travel cost is equal to the tour's duration.

The described problem is NP-hard because it is an extension of the traveling salesman problem with pickup and delivery (TSPPD), which itself is an extension of the traveling salesman problem (TSP). In contrast to the TSPPD and the TSP, in our problem, it is not necessary to visit all the requests.

The main contributions of this paper are summarized as follows. First, we introduce a new model which extends the classical TSPPD by having time-dependent travel times and the option to reject requests. Secondly, we propose an exact solution method for this problem by developing a tailored labeling algorithm in which novel and strong dominance criteria are used. Finally, a restricted dynamic programing heuristic is proposed with a high solution quality and lower computation times than the labeling algorithm.

The remainder of the paper is structured as follows. Section 2 provides a brief review of related existing work. Section 3 defines the problem and introduces a mathematical formulation of the problem. In Section 4, we present the tailored labeling algorithm and in Section 5 the restricted dynamic programing heuristic is introduced. Finally, computational results are reported in Section 6, followed by conclusions in Section 7 .

\section{Literature review}

There are three classes of problems closely related to the problem studied in this paper: the traveling salesman problem with pickup and delivery (TSPPD), the time-dependent vehicle routing problem (TDVRP) and the traveling salesman problem with profits (TSP with profits).

\subsection{The traveling salesman problem with pickup and delivery (TSPPD)}

Our problem extends the TSPPD by considering time-dependent travel times. The TSPPD is firstly introduced by Ruland and Rodin (1997) and is proven to be of great use in applications like dial-aride systems and courier services. Although the pickup and delivery problem (PDP), in which the TSPPD can appear as a subproblem, is extensively studied in the literature, only limited research focuses on the TSPPD.

Currently, the most popular methodology for solving the TSPPD is branch-and-cut. Ruland (1994) and Ruland and Rodin
(1997) considered the undirected case of this problem and developed four classes of valid inequalities that are embedded in a branch-and-cut algorithm. The algorithm is tested on instances with up to 15 pickup and delivery requests. Recently, Dumitrescu, Ropke, and Cordeau (2010) studied the same problem, the authors analyzed its polyhedral structure and proposed new valid inequalities that are shown to be facets for the TSPPD polytopes. Their algorithm is capable of solving instances with up to 35 pickup and delivery requests to optimality.

The TSPPD appears as pricing problem for the PDP and is in that situation usually named as the elementary shortest path problem with time windows, capacity and pickup and delivery (ESPPTWCPD). Sol (1994), Sigurd and Pisinger (2004) and Ropke, Cordeau, and Laporte (2009) presented labeling algorithms with several different dominance rules to solve this problem to optimality.

\subsection{The time-dependent vehicle routing problem with time windows (TDVRP)}

Another related problem is the TDVRP. Although the TDVRP has attracted the attention of many researchers, literature on this subject remains scarce. The pioneering work is done by Malandraki and Daskin (1992) and Malandraki and Dial (1996). In these papers mixed integer linear programs and several heuristics to solve the problem are proposed. The First-In-First-Out (FIFO) property, which implies that for every arc a later departure time results in a later (or equal) arrival time, is an intuitive and desirable property for time dependent routing problems. Ichoua, Gendreau, and Potvin (2003) and Dabia, Ropke, van Woensel, and de Kok (2013) considered the TDVRP with travel time variability modeled by "constant speed" time periods, which ensures the FIFO property. The idea of constant speed time periods is adopted in our problem as well.

Due to the complexity of the time-dependent problem, most of the existing algorithms are based on heuristics. In van Woensel, Kerbache, Peremans, and Vandaele (2008) a tabu search heuristic is used to solve the capacitated vehicle routing problem with time dependent travel times. An approximation based on queueing theory and the number of vehicles on a link is used to determine travel speeds. Donati, Montemanni, Casagrande, Rizzolo, and Gambardella (2008) developed a multi-ant colony system for the TDVRP and Ibaraki etal. (2008) proposed an iterated local search heuristic for the time-dependent vehicle routing problem with time windows (TDVRPTW). Recently, Dabia etal. (2013) 
Table 1

Parameters.

\begin{tabular}{ll}
\hline Notation & Definition \\
\hline$R=\{1, \ldots, n\}$ & Set of requests \\
$N$ & Set of nodes \\
$A$ & Set of arcs \\
$N_{P}$ & Set of pickup nodes \\
$N_{D}$ & Set of delivery nodes \\
$(i, n+i)$ & A transportation request $R_{i}$ \\
$r_{i}$ & Profit of request $R_{i}$ \\
$q_{i}$ & Demand of request $R_{i}$ \\
$Q$ & Carrying capacity of the vehicle \\
{$\left[e_{i}, l_{i}\right]$} & Time window of node $i$ \\
$s_{i}$ & Service time at node $i$ \\
$t_{i}$ & Departure time from node $i$ \\
$\tau_{i j}\left(t_{i}\right)$ & Travel time from node $i$ to node $j$ with departure time $t_{i}$ at node $i$ \\
\hline
\end{tabular}

developed a branch-and-price algorithm for the TDVRPTW, where a tailored labeling algorithm is presented to solve the timedependent shortest path problem with resource constraint (TDSP$\mathrm{PRC})$, which is the pricing problem in the algorithm.

\subsection{The traveling salesman problem with profits (TSP with profits)}

The proposed problem extends the traveling salesman problem with profits (TSP with profits), in which profits are associated with each request and the overall goal is to find the shortest tour with the maximum collected profits. This means that in contrast to the original TSP, not all nodes have to be visited. In comparison with our study, it does not include time dependency and requests with pickup and delivery nodes.

According to Feillet, Dejax, and Gendreau (2005), TSPs with profits can be categorized into three generic problems depending on the way the terms profits and travel time are addressed in the objective function and constraints. They can be classified in the following categories: the profitable tour problem (PTP), the prizecollecting traveling salesman problem (PCTSP), and the orienteering problem (OP). Dell' Amico, Maffioli, and Varbrand (1995) studied the profitable tour problem (PTP) where both the profits and the travel time are combined in the objective function. Our study builds upon the PTP by having the profits and the travel time in the objective as well. In the prize-collecting TSP (PCTSP) the objective is similar to PTP but a constraint is added to ensure that a minimum amount of profits is collected within the tour. In the original definition of the PCTSP by Balas (1989) there were also penalty values for unvisited nodes within the objective function. An abundant number of publications is devoted to the orienteering problem (OP), which aims to maximize the collected profits subject to a constraint on the maximum allowed tour length. This problem is also known as the selective traveling salesman problem (Laporte \& Martello, 1990).

A variant of the OP is the time-dependent orienteering problem (TDOP) which includes time-dependent travel times. Fomin and Lingas (2002) provided a $(2+\epsilon)$-approximation algorithm for the TDOP which runs in polynomial time if the ratio between the minimum and maximum travel time between any two sites is constant. Li (2011) designed a novel dynamic labeling algorithm for the TDOP in which time is measured in discrete units. Therefore, the FIFO property may not be satisfied in their model. Verbeeck, Aghezzaf, and Vansteenwegen (2014) provided a fast solution method for the TDOP based on an ant colony optimization algorithm. Recently, Verbeeck, Vansteenwegen, and Aghezzaf (2016) presented an ant colony optimization based algorithm for the stochastic variant of the TDOP, which is addressed as the stochastic time-dependent orienteering problem with time windows. For more details about the OP and its variants, readers are referred to Vansteenwegen, Souffriau, and Oudheusden (2011) and Gunawan, Lau, and Vansteenwegen (2016).

To the best of our knowledge, no literature is found that handles a combination of precedence in pickup and delivery, profitmaximizing selection and time-dependent travel time routing cost minimization at the same time. Thus, in this study, we introduce the time-dependent capacitated profitable tour problem with time windows and precedence constraints, which takes care of these three challenges simultaneously. Moreover, both exact and heuristic methods are proposed to solve this problem.

\section{Problem description and mathematical formulation}

In this section, we first define the problem and introduce the notation used throughout the paper. Afterwards, we present a mathematical formulation for the problem.

\subsection{Problem definition}

The time-dependent capacitated profitable tour problem with time windows and precedence constraints is defined as follows. We consider a set of $n$ requests $R_{1}, \ldots, R_{n}$, where $R_{i}(i=1, \ldots, n)$ is associated with the pickup node $i$ and the corresponding delivery node $n+i$. Let $G=(N, A)$ be a directed graph, where $N=$ $\{0,1, \ldots, 2 n+1\}$ is the set of all nodes, and 0 and $2 n+1$ represent the origin and destination depot of the vehicle. We define the subsets $N_{P}=\{1, \ldots, n\}$ and $N_{D}=\{n+1, \ldots, 2 n\}$ as the pickup and delivery nodes, respectively. With each pickup node $i \in N_{P}$ a profit $r_{i}$ and a load $q_{i}$ are associated, and with each delivery node a load $q_{n+i}$ is associated. For the requests, it must hold that $q_{i}=-q_{n+i}$. There is no inventory at the depots and therefore $q_{0}=q_{2 n+1}=0$. To serve the requests we have one vehicle available with limited capacity $Q$.

A hard time window $\left[e_{j}, l_{j}\right]$ is associated with each node $j \in N_{P} \cup N_{D}$, where $e_{j}$ and $l_{j}$ represent the earliest and latest time, respectively, at which the service at node $j$ may start. The service time is denoted by $s_{j}$. A vehicle needs to wait until time $e_{j}$, if it is arriving at node $j$ before time $e_{j}$; and arriving later than $l_{j}$ is not allowed. We denote $\left[e_{0}, l_{0}\right],\left[e_{2 n+1}, l_{2 n+1}\right]$ as the time windows of the origin and the destination depot, respectively. Without loss of generality, we assume that $e_{0}=0$ and $s_{0}=s_{2 n+1}=0$.

Let $\tau_{i j}\left(t_{i}\right)$ denote the travel time from node $i$ to node $j$, which depends on the departure time $t_{i}$ at node $i$. Then, we can define the set of feasible arcs as $A=\left\{(i, j) \in N \times N: i \neq j\right.$ and $e_{i}+s_{i}+$ $\left.\tau_{i j}\left(e_{i}+s_{i}\right) \leq l_{j}\right\}$. This means that an arc from node $i$ to node $j$ is only included if it is possible to go from node $i$ to $j$ while respecting the time windows of both nodes.

The notation is summarized in Table 1.

The planning horizon is divided into several time periods. Each $\operatorname{arc}(i, j) \in A$ has a speed profile associated with it, which consists of 

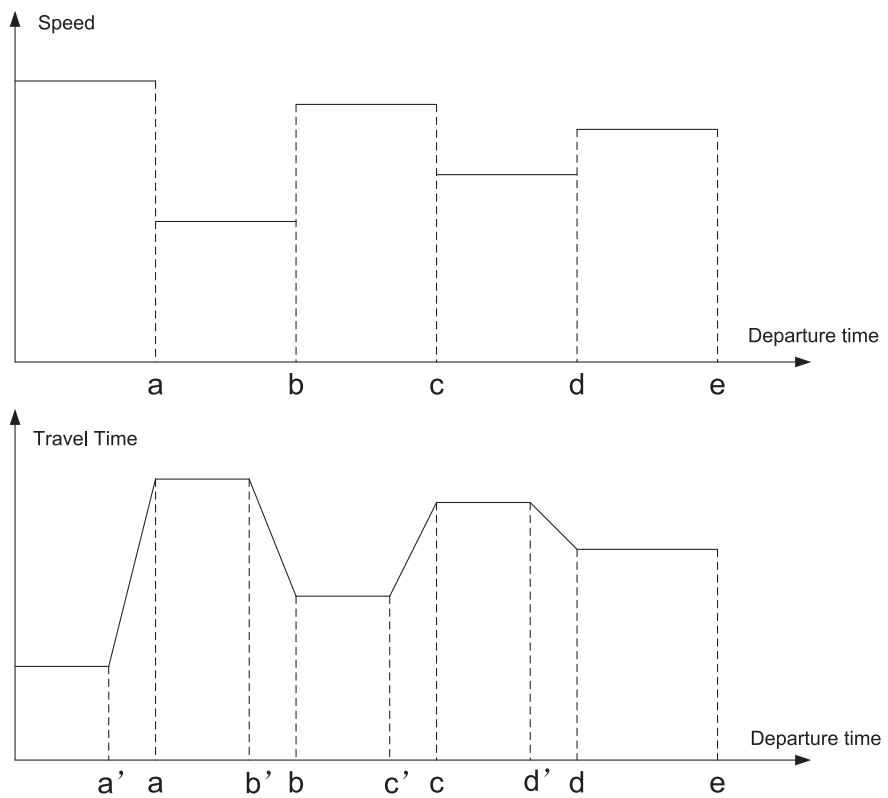

Fig. 2. Speed and travel time functions.

a constant speed within each time period. By using those stepwise speed functions, the FIFO property holds for every arc in the graph $G$ (i.e., a later departure always leads to a later arrival and therefore overtaking will not occur). The speed profiles can be different for each arc.

Fig. 2 depicts a speed profile and the corresponding travel time function for some arc $(i, j)$. Using the idea described in Ichoua et al. (2003), we denote the points $a, b, c, d$, and $e$ where the speed changes as speed breakpoints. There are also travel time breakpoints in the travel time function. These are the departure times which ensure an arrival at node $j$ exactly at the time of a speed breakpoint (e.g., $a^{\prime}$ is the departure time at node $i$ to arrive at node $j$ at time $a)$.

The travel time function is piecewise linear and can be represented by the breakpoints values. Note that in case of timedependent travel times, the triangle inequality does not necessarily hold. Intuitively, when the direct link between node $h$ and $l$ is heavily congested, we may reach destination node $l$ earlier by taking a diverted routed (i.e., via one or several other nodes) than by the direct link from node $h$.

Because of the FIFO property of the travel time functions, a later departure at the depot 0 always results in a later arrival time at node $i$. Therefore, if a path is infeasible for a certain departure time $t_{0}$ at the origin depot (i.e., a time window of a node in the path is violated), it will also be infeasible for any departure time $t^{\prime} \geq t_{0}$ at the origin depot. Given a path $p=\left(v_{0}, v_{1}, \ldots, v_{k}\right)$ with $v_{0}=0$ and $v_{i}$ being the node at position $i$ in the path $p$, we define $\delta_{v_{i}}^{p}(t)$ as the ready time function at node $v_{i}$ in path $p$ given a departure time $t$ at node 0 . This ready time function is nondecreasing in $t$ and can be calculated recursively for each node in the path as follows:

$\delta_{v_{i}}^{p}(t)= \begin{cases}t & \text { if } i=0, \\ \max \left\{e_{v_{i}}+s_{v_{i}}, \delta_{v_{i-1}}^{p}(t)\right. & \\ \left.+\tau_{v_{i-1}, v_{i}}\left(\delta_{v_{i-1}}^{p}(t)\right)+s_{v_{i}}\right\} & \text { otherwise. }\end{cases}$

The ready time function is piecewise linear and this means that we can represent the ready time function by using the ready time function breakpoints. These are the breakpoints of the ready time function of the predecessor node, breakpoints of the travel time function, and the boundary values of the time window of node $v_{i}$.

The duration of the path given a departure time $t$ at node 0 can be calculated as $\delta_{v_{k}}^{p}(t)-t$, which is again a piecewise linear function. In this problem we minimize the total duration of the selected tour instead of the sum of the arc cost. As the duration is a piecewise linear function of the departure time, it is clear that the minimum duration of a tour can be computed by only considering the breakpoints of the ready time function.

\subsection{Mathematical formulation}

For every arc $(i, j) \in A$, we denote $T_{i j}$ as the set of time periods of the corresponding travel time function $\tau_{i j}\left(t_{i}\right)$. A time period $T_{m} \in T_{i j}$, is defined by two consecutive travel time breakpoints, $T_{m}=\left[w_{m}, w_{m+1}\right]$. As $\tau_{i j}\left(t_{i}\right)$ is linear in each time period, using $w_{m}$, $w_{m+1}, \tau_{i j}\left(w_{m}\right)$ and $\tau_{i j}\left(w_{m+1}\right)$, we can easily calculate the corresponding slope $\theta_{m}$ and its intersection $\eta_{m}$ with the $y$-axis. Therefore

$\tau_{i j}\left(t_{i}\right)=\theta_{m} t_{i}+\eta_{m} . \quad \forall t_{i} \in T_{m}$

Furthermore, let $x_{i j}^{m}$ be a binary variable that takes value 1 if and only if the vehicle traverses the $\operatorname{arc}(i, j) \in A$ with a departure time in time period $m$. A variable $t_{i j}^{m}$ is introduced to denote this departure time of traveling from $i$ to $j$ in time period $T_{m}$. This means that $t_{i j}^{m}$ is such that

$t_{i j}^{m}= \begin{cases}t_{i} & \text { if } x_{i j}^{m}=1 \\ 0 & \text { otherwise }\end{cases}$

Consequently, when traveling from $i$ to $j$, we have that:

$t_{i}=\sum_{j \in N \backslash\{0\}} \sum_{m=0}^{\left|T_{i j}\right|} t_{i j}^{m}$

It means that the travel time function $\tau_{i j}\left(t_{i}\right)$ of $\operatorname{arc}(i, j)$ at node $i$ can be written as:

$\tau_{i j}\left(t_{i}\right)=\sum_{m=0}^{\left|T_{i j}\right|}\left(\theta_{m} t_{i j}^{m}+\eta_{m} x_{i j}^{m}\right)$.

Let $y_{i}$ be a binary variable that equals 1 if and only if node $i \in N_{P} \cup N_{D}$ is visited. Furthermore, let $Q_{i}, i \in N$ be a nonnegative integer variable that is the load of the vehicle upon departure from node $i$. Then the mixed integer programing formulation is given as follows:

$\max \sum_{j \in N_{P}} r_{j} y_{j}-\left(t_{2 n+1}-t_{0}\right)$

subject to

$\sum_{j \in N_{P}} \sum_{m=0}^{\left|T_{0 j}\right|} x_{0 j}^{m}=1$

$\sum_{i \in N_{D}} \sum_{m=0}^{\left|T_{i, 2 n+1}\right|} x_{i, 2 n+1}^{m}=1$

$\sum_{i \in N \backslash\{2 n+1\}} \sum_{m=0}^{\left|T_{i j}\right|} x_{i j}^{m}=y_{j} \quad \forall j \in N \backslash\{0\}$

$\sum_{i \in N \backslash\{2 n+1\}} \sum_{m=0}^{\left|T_{i k}\right|} x_{i k}^{m}-\sum_{j \in N \backslash\{0\}} \sum_{m=0}^{\left|T_{k j}\right|} x_{k j}^{m}=0 \quad \forall k \in N \backslash\{0,2 n+1\}$

$\sum_{j \in N \backslash\{0\}} \sum_{m=0}^{\left|T_{i j}\right|} x_{i j}^{m}-\sum_{j \in N \backslash\{0\}} \sum_{m=0}^{\left|T_{n+i, j}\right|} x_{n+i, j}^{m}=0 \quad \forall i \in N_{P}$ 
$t_{j} \geq\left(1+\theta_{m}\right) t_{i j}^{m}+\eta_{m} x_{i j}^{m}+s_{j} x_{i j}^{m} \quad \forall i \in N \backslash\{2 n+1\}, j \in N \backslash\{0\}$

$Q_{i}+q_{j} \leq Q_{j}+M\left(1-x_{i j}^{m}\right)$

$\forall i, j \in N, \forall m, m+1 \in\left|T_{i j}\right|$

$t_{n+i} \geq t_{i}$

$\forall i \in N_{P}$

$t_{i}=\sum_{j \in N \backslash\{0\}} \sum_{m=0}^{\left|T_{i j}\right|} t_{i j}^{m}$

$\forall i \in N \backslash\{2 n+1\}$

$w_{m} x_{i j}^{m} \leq t_{i j}^{m} \leq w_{m+1} x_{i j}^{m}$

$\forall i, j \in N, \forall m, m+1 \in\left|T_{i j}\right|$

$e_{i} y_{i} \leq t_{i} \leq l_{i} y_{i}$

$\forall i \in N_{P} \cup N_{D}$

$\max \left\{0, q_{i}\right\} \leq Q_{i} \leq \min \left\{Q, Q+q_{i}\right\} \quad \forall i \in N$

$x_{i j}^{m}, y_{i} \in\{0,1\}$

$\forall i, j \in N, \forall m \in\left|T_{i j}\right|$

The objective function (6) aims to find a tour that maximizes the collected profits minus the total traveling duration. Constraints (7)-(8) guarantee that the path starts at the origin depot 0 and ends at the destination depot $2 n+1$. Constraints (9) guarantee that every node, except the nodes representing the start and end depots, is visited at most once. Constraints (10) keep the flow conservation. Constraints (11) ensure that it is not possible to visit only the pickup node or only the delivery node of a certain request. Constraints (12) guarantee that the departure time at a node in the route is larger or equal than the sum of the departure time from the previous node, the travel time between these two nodes and the required service time. Constraints (13) determine the consistency of the load variables. Precedence constraints (14) ensure that for each request $i$, the pickup node is visited before the delivery node. Constraints (15) are formulated as mentioned before in (4). Constraints (16) and (17) force the departure time of each request to be in the given time period and the given time window. Finally, Constraints (18) ensure that the load in the vehicle is never larger than the capacity of the vehicle.

Due to the computational inefficiency of solving large-scale instances with a commercial ILP solver, we develop a tailored labeling algorithm to solve this problem.

\section{A tailored labeling algorithm}

In order to solve our problem, we introduce a new exact dynamic programing algorithm, that is named as the tailored labeling algorithm. Ropke etal. (2009) developed a labeling algorithm to solve the pickup and delivery problems with time windows (PDPTW). However, this time-independent algorithm is only efficient if the triangle inequality holds. More recently, Dabia etal. (2013) proposed another labeling algorithm for the timedependent vehicle routing problem with time windows. Their algorithm has great potential for the time-dependent routing problem without precedence constraints.

In our situation the triangle inequality does not necessarily hold due to the time dependent travel times and furthermore precedence constraints are present. Therefore, we need to develop a new algorithm. Note that the proposed algorithm can be generalized to solve other time-dependent routing problems with precedence constraints.
The algorithm starts generating labels from the depot 0 . It progressively extends all feasible labels until they reach the end depot $2 n+1$. Moreover, to speed up our tailored labeling algorithm, instead of starting the label extension only from the origin depot 0 in a forward direction, we simultaneously generate labels in backward direction from the destination depot to its predecessors as well. Where both forward labels and backward labels are extended to some time $t_{m}$ (e.g., the middle of the planning horizon) but not further. At the end, complete paths are generated by merging the partial paths of forward and backward labels. All complete paths are evaluated and the path with the best objective function value is the optimal path. This bidirectional approach has shown great potential for improving the running time of related resource constrained shortest path problems (see, e.g., Righini and Salani, 2006; Dabia et al., 2013).

The forward labeling algorithm is introduced in Section 4.1, followed by the backward labeling algorithm in Section 4.2. If labels are dominated by the criterion introduced in Sections 4.1.3 and 4.2 .3 , they are removed from the list. Note that if in the procedure none of the labels are dominated, this algorithm is equal to the complete enumeration of all feasible paths. Therefore the dominance criterion is very important. Finally, we discuss the way to merge the partial paths of forward and backward labels in Section 4.3.

\subsection{The forward labeling algorithm}

In the forward labeling algorithm we start generating labels from the start depot. The definition of a forward label is discussed in Section 4.1.1. The labels are extended if the extension is feasible as discussed in Section 4.1.2. The dominance criterion for forward labels is discussed in Section 4.1.3.

\subsubsection{Forward label}

For each forward label $L_{f}$, we use the following notation:

$p\left(L_{f}\right) \quad$ The partial path of label $L_{f}$.

$v\left(L_{f}\right) * \quad$ The last node visited on the partial path $p\left(L_{f}\right)$.

$L^{-1}\left(L_{f}\right) *$ The parent label from which $L_{f}$ originates by extending it with $v\left(L_{f}\right)$.

$O\left(L_{f}\right)$ * The set of incomplete requests in $p\left(L_{f}\right)$, i.e., the pickup node is visited but not the delivery node.

$U\left(L_{f}\right)$ * The set of requests for which the pickup nodes are already visited along the partial path $p\left(L_{f}\right)$. It contains both the complete and the incomplete requests. Therefore, $O\left(L_{f}\right) \subseteq U\left(L_{f}\right)$.

$P\left(L_{f}\right) \quad$ The set of pickup nodes not visited in $p\left(L_{f}\right)$, i.e., $j \in N_{P}$ and $R_{j} \notin U\left(L_{f}\right)$.

$D\left(L_{f}\right) \quad$ The set of delivery nodes of incomplete requests in $p\left(L_{f}\right)$, i.e., $j \in N_{D}$ and $R_{j-n} \in O\left(L_{f}\right)$.

$q\left(L_{f}\right) * \quad$ The load of the vehicle after visiting node $v\left(L_{f}\right)$.

$\delta_{L_{f}}(t) * \quad$ The piecewise linear function that represents the ready time at $v\left(L_{f}\right)$ if the vehicle departed at the origin depot at $t$ and reached $v\left(L_{f}\right)$ through partial path $p\left(L_{f}\right)$. Moreover, $\delta_{L_{f}}(0)$ is the earliest ready time at $v\left(L_{f}\right)$ since the earliest departure time at the origin depot is 0 .

$r\left(L_{f}\right) * \quad$ The overall profits collected by serving the requests visited on the partial path $p\left(L_{f}\right)$.

Only the items marked with a * are stored in the label. The set $D\left(L_{f}\right)$ and $P\left(L_{f}\right)$ can be deduced from the sets $O\left(L_{f}\right)$ and $U\left(L_{f}\right)$. Furthermore, the partial path can be deduced from iteratively checking the last node visited in the parent label of which the this label was an extension. 
(a)

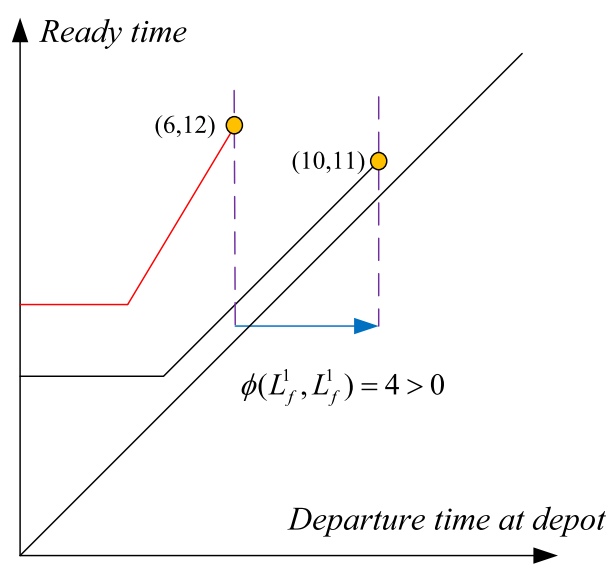

(b)

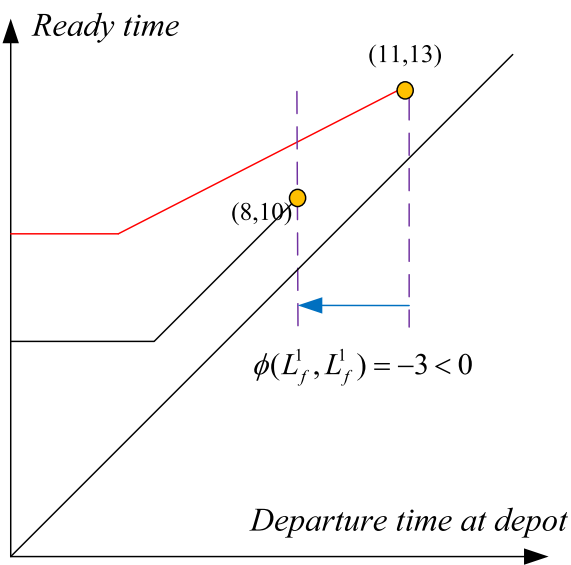

(c)

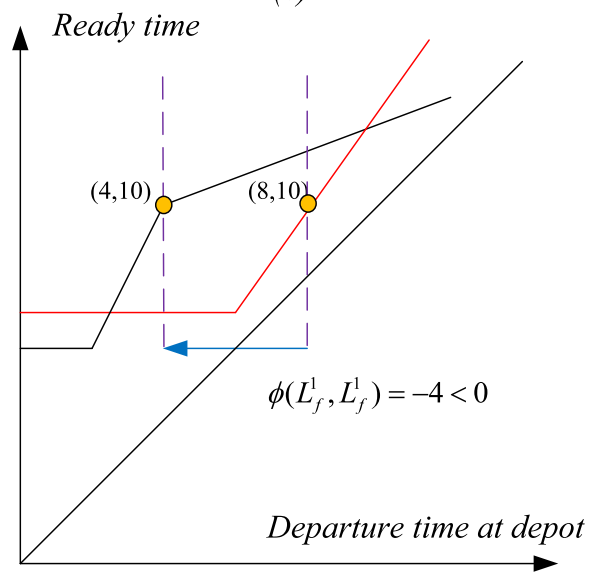

Ready time for label $L_{f}^{1}$

Ready time for label $L_{f}^{2}$

Fig. 3. Illustration of $\phi\left(L_{f}^{1}, L_{f}^{2}\right)$.

\subsubsection{Label extension}

We extend a label $L_{f}^{\prime}$ along an $\operatorname{arc}\left(v\left(L_{f}^{\prime}\right), j\right)$, only when the extension is feasible in terms of time windows and capacity. First, the following two conditions should be met:

$\delta_{L_{f}^{\prime}}(0)+\tau_{v\left(L_{f}^{\prime}\right), j}\left(\delta_{L_{f}^{\prime}}(0)\right)+s_{j} \leq \min \left\{t_{m}, l_{j}+s_{j}\right\} \wedge j \in N \backslash\{0\}$

$q\left(L_{f}\right)+q_{j} \leq Q \wedge j \in N \backslash\{0\}$

Condition (20) ensures that an extension to node $j$ can only be performed if node $j$ can be reached within its time window and guarantees that the extension is stopped before $t_{m}$ is exceeded. Condition (21) ensures that an extension to node $j$ is only possible if there is enough capacity to deal with the load of node $j$.

Secondly, $L_{f}^{\prime}$ and $j$ must also satisfy one of the following three conditions:

$j \notin U\left(L_{f}^{\prime}\right) \wedge j \in N_{P}$

$j-n \in O\left(L_{f}^{\prime}\right) \wedge j \in N_{D}$

$O\left(L_{f}^{\prime}\right)=\emptyset \wedge j=2 n+1$

Condition (22) states that $j$ should not have been visited before, if it is a pickup node. Condition (23) indicates that if $j$ is a delivery node, the corresponding pickup node should have been visited already. The last condition, Condition (24), states that if $j$ is the end depot then all visited requests should have been completed. In the presence of those conditions, only elementary paths that satisfy precedence constraint (11) are generated.

At last, we need to check that all delivery nodes of requests for which the pickup node is already visited in $p\left(L_{f}^{\prime}\right)$ can still be reached. In case the triangle inequality holds, a node is unreachable if traversing the direct arc from $j$ to this node is not possible by capacity, time window or precedence constraints. However, time-dependent travel times cannot guarantee the triangle inequality. Therefore, a node that is unreachable via the direct arc from node $j$ by the time window constraints might still be reachable indirectly via a diverted route. First, we need to know the earliest ready time at node $j$ after following partial path $p\left(L_{f}^{\prime}\right)$ before visiting node $j$, which will be denoted by $t_{r}\left(L_{f}^{\prime}, j\right)$. It holds that
$t_{r}\left(L_{f}^{\prime}, j\right)=\max \left\{e_{j}+s_{j}, \delta_{L_{f}^{\prime}}(0)+\tau_{L_{f}^{\prime}, j}\left(\delta_{L_{f}^{\prime}}(0)\right)+s_{j}\right\}$. Then, we need for any unvisited node $k$ the earliest arrival time given that the vehicle visits node $j$ and $k$ consecutively after partial path $p\left(L_{f}^{\prime}\right)$, which can be computed by $t_{r}\left(L_{f}^{\prime}, j\right)+\tau_{j k}\left(t_{r}\left(L_{f}^{\prime}, j\right)\right)$. Finally, the earliest possible time the vehicle could reach a node after node $j$ is given by $t_{e}\left(L_{f}^{\prime}, j\right)=\min _{k \in P\left(L_{f}^{\prime}\right) \cup D\left(L_{f}^{\prime}\right)}\left\{t_{r}\left(L_{f}^{\prime}, j\right)+\tau_{j k}\left(t_{r}\left(L_{f}^{\prime}, j\right)\right)\right\}$. This means that any node $k$ with the latest allowed arrival time $l_{k}$ earlier than this time (i.e., $l_{k}<t_{e}\left(L_{f}^{\prime}, j\right)$ ) is unreachable from $j$, also in an indirect way as no node could be reached before $t_{e}\left(L_{f}^{\prime}, j\right)$. If a delivery node $k$ is unreachable after $j$ and its corresponding pickup node is already visited (i.e., $k-n \in O\left(L_{f}^{\prime}\right)$ ), the extension to $j$ is not feasible as the picked up item cannot be delivered anymore. Therefore, as stated in condition (25), all delivery nodes of requests of which the pickup node is already visited in $p\left(L_{f}^{\prime}\right)$ should still be reachable to make sure that extending label $L_{f}^{\prime}$ to $j$ is feasible. Note that this test can be done quickly, but we might fail to find all unreachable delivery nodes.

$l_{k} \geq t_{e}\left(L_{f}^{\prime}, j\right) \quad \forall k \in D\left(L_{f}^{\prime}\right): k \neq j$

If the extension along the arc $\left(v\left(L_{f}^{\prime}\right), j\right)$ is feasible according to all described conditions, then a new label $L_{f}$ is created. The information in label $L_{f}$ is updated as follows:

$L^{-1}\left(L_{f}\right)=L_{f}^{\prime}$

$v\left(L_{f}\right)=j$

$\delta_{L_{f}}(t)=\max \left\{e_{j}+s_{j}, \delta_{L_{f}^{\prime}}(t)+\tau_{L_{f}^{\prime}, j}\left(\delta_{L_{f}^{\prime}}(t)\right)+s_{j}\right\}$

$q\left(L_{f}\right)=q\left(L_{f}^{\prime}\right)+q_{j}$

$r\left(L_{f}\right)= \begin{cases}r\left(L_{f}^{\prime}\right)+r_{j} & \text { if } j \in N_{P} \\ r\left(L_{f}^{\prime}\right) & \text { otherwise }\end{cases}$

$O\left(L_{f}\right)= \begin{cases}O\left(L_{f}^{\prime}\right) \cup\{j\} & \text { if } j \in N_{P} \\ O\left(L_{f}^{\prime}\right) \backslash\{j-n\} & \text { if } j \in N_{D}\end{cases}$ 
$U\left(L_{f}\right)= \begin{cases}U\left(L_{f}^{\prime}\right) \cup\{j\} & \text { if } j \in N_{P}, \\ U\left(L_{f}^{\prime}\right) & \text { otherwise }\end{cases}$

Eqs. (26) -(30) set the last visited node, the ready time function, the load, and the collected profits of the new label, respectively. Eq. (31) updates the set of incomplete requests $O\left(L_{f}\right)$ and Eq. (32) updates the set of visited pickup nodes $U\left(L_{f}\right)$.

\subsubsection{Label dominance}

Let $\operatorname{dom}\left(L_{f}\right)$ and $\operatorname{img}\left(L_{f}\right)$ be the domain and image of the ready time function $\delta_{L_{f}}(t)$, respectively. If the partial path is feasible, a departure at time 0 from the origin depot is always feasible. Therefore, $\operatorname{dom}\left(L_{f}\right)$ is always of the form $[0, t]$ for some $t \geq 0$. When $v\left(L_{f}\right)=2 n+1$, the objective function value of the path corresponding to $L_{f}$ is:

$\operatorname{obj}\left(L_{f}\right)=r\left(L_{f}\right)-\min _{t \in \operatorname{dom}\left(L_{f}\right)}\left\{\delta_{L_{f}}(t)-t\right\}$

In the labeling algorithm, all possible extensions are processed and stored for each label. However, the number of labels that can be processed is typically very large and computationally expensive. Therefore, a dominance test is established between pairs of labels that have the same last visited node. The number of labels is reduced by only storing the non-dominated labels. Before the dominance criterion is introduced some definitions need to be provided.

First, similar to the idea of Feillet, Dejax, and Gendreau (2004), we introduce for every label $L_{f}$ the set $\widetilde{U}\left(L_{f}\right)$ which extends $U\left(L_{f}\right)$ by adding requests of which the pickup node is unreachable from $v\left(L_{f}\right)$. Similar to the discussion in Section 4.1.2 it can be derived that the earliest possible time the vehicle could reach a pickup node after $v\left(L_{f}\right)$ is given by $t_{e}\left(L_{f}\right)=\min _{j \in P\left(L_{f}\right) \cup D\left(L_{f}\right)}\left\{\delta_{L_{f}}(0)+\right.$ $\left.\tau_{v\left(L_{f}\right) j}\left(\delta_{L_{f}}(0)\right)\right\}$. This means that any pickup node $j$ with the latest allowed arrival time $l_{j}$ earlier than this time (i.e., $l_{j}<t_{e}\left(L_{f}\right)$ ) is unreachable from $v\left(L_{f}\right)$. Therefore, the corresponding request cannot be served anymore and can be added to the set $\widetilde{U}\left(L_{f}\right)$. Note that the check $l_{j}<t_{e}\left(L_{f}\right)$ does not guarantee to find all unreachable pick up nodes.

Secondly, we define the interval

$I \subseteq\left(-\infty, \max \left(\operatorname{dom}\left(L_{f}^{1}\right)\right)-\max \left(\operatorname{dom}\left(L_{f}^{2}\right)\right)\right)$.

Based on $I$, we also define a real number $\phi\left(L_{f}^{1}, L_{f}^{2}\right)$,

$$
\begin{aligned}
\phi\left(L_{f}^{1}, L_{f}^{2}\right)= & \max \left\{x \in I: \delta_{L_{f}^{1}}(\max \{0, t+x\}) \leq \delta_{L_{f}^{2}}(t),\right. \\
& \left.\forall t \in \operatorname{dom}\left(L_{f}^{2}\right)\right\} .
\end{aligned}
$$

When $\phi\left(L_{f}^{1}, L_{f}^{2}\right)$ is positive, it indicates that the vehicle can depart at maximum $\phi\left(L_{f}^{1}, L_{f}^{2}\right)$ time units later when traversing partial path $p\left(L_{f}^{1}\right)$ instead of traversing $p\left(L_{f}^{2}\right)$, to still reach node $v\left(L_{f}^{1}\right)$ earlier via path $p\left(L_{f}^{1}\right)$ then via partial path $p\left(L_{f}^{2}\right)$. When it is negative, it indicates that the vehicle should depart at least $\phi\left(L_{f}^{1}, L_{f}^{2}\right)$ time units earlier when traversing partial path $p\left(L_{f}^{1}\right)$ instead of traversing $p\left(L_{f}^{2}\right)$, to be able to reach node $v\left(L_{f}^{1}\right)$ earlier via path $p\left(L_{f}^{1}\right)$ then via partial path $p\left(L_{f}^{2}\right)$.

In Fig. 3, we depict several simple examples: if there is no intersection between labels $L_{f}^{1}$ and $L_{f}^{2}, \phi\left(L_{f}^{1}, L_{f}^{2}\right)$ is positive when $\max \left(\operatorname{dom}\left(L_{f}^{1}\right)\right)>\max \left(\operatorname{dom}\left(L_{f}^{2}\right)\right)$ (see Fig. 3(a)), or negative when $\max \left(\operatorname{dom}\left(L_{f}^{1}\right)\right)<\max \left(\operatorname{dom}\left(L_{f}^{2}\right)\right)$ (see Fig. 3(b)). Otherwise, $\phi\left(L_{f}^{1}, L_{f}^{2}\right)$ can only be negative (see Fig. 3(c)). lows:

Finally, the dominance test is stated in Proposition 4.1 as fol-
Proposition 4.1. Label $L_{f}^{2}$ is dominated by label $L_{f}^{1}$ if

$$
\begin{aligned}
& \text { 1. } v\left(L_{f}^{1}\right)=v\left(L_{f}^{2}\right) \\
& \text { 2. } U\left(L_{f}^{1}\right) \subseteq \widetilde{U}\left(L_{f}^{2}\right) \\
& \text { 3. } O\left(L_{f}^{1}\right)=O\left(L_{f}^{2}\right) \\
& \text { 4. } \delta_{L_{f}^{1}}(0) \leq \delta_{L_{f}^{2}}(0) \\
& \text { 5. } r\left(L_{f}^{1}\right) \geq r\left(L_{f}^{2}\right)-\phi\left(L_{f}^{1}, L_{f}^{2}\right) \\
& \text { 6. } q\left(L_{f}^{1}\right) \leq q\left(L_{f}^{2}\right)
\end{aligned}
$$

Proof of Proposition 4.1. Consider two labels $L_{f}^{1}$ and $L_{f}^{2}$ that satisfy the six conditions in Proposition 4.1. We need to show that (i) any feasible extension $L$ that extends $p\left(L_{f}^{2}\right)$ to $2 n+1$ is also a feasible extension for $p\left(L_{f}^{1}\right)$ to $2 n+1$ and (ii) that for all these feasible extensions $L$ it holds that $\operatorname{obj}\left(L_{f}^{1} \oplus L\right) \geq \operatorname{obj}\left(L_{f}^{2} \oplus L\right)$, where $L_{f} \oplus L$ is the label resulting from extending $L_{f}$ with $L$.

With regards to point (i), first, capacity will not be violated along the path $p\left(L_{f}^{1} \oplus L\right)$ as it was not violated on path $p\left(L_{f}^{2} \oplus L\right)$ and by condition 6 it holds that $q\left(L_{f}^{1}\right) \leq q\left(L_{f}^{2}\right)$. Secondly, the path $p\left(L_{f}^{1} \oplus L\right)$ is elementary. By conditions 2 and 3 , all nodes visited in $p\left(L_{f}^{1}\right)$ are either nodes visited in $p\left(L_{f}^{2}\right)$ or nodes which could not be reached by any extension of label $L_{f}^{2}$ (i.e., the nodes of requests included in $\left.\widetilde{U}\left(L_{f}^{2}\right) \backslash U\left(L_{f}^{2}\right)\right)$. A feasible extension of $L_{f}^{2}$ cannot contain any node visited along path $p\left(L_{f}^{2}\right)$ or node which is unreachable from label $L_{f}^{2}$. Therefore, all nodes visited along path $p\left(L_{f}^{1}\right)$ are not visited in $L$, so path $p\left(L_{f}^{1} \oplus L\right)$ is elementary as well. Third, there exists a departure time for path $p\left(L_{f}^{1} \oplus L\right)$ which does not violate time windows. As $L$ is a feasible extension of $L_{f}^{2}$ it means that there is a departure time at $v\left(L_{f}^{1}\right)$ after $\delta_{L_{f}^{2}}(0)$ making sure that all nodes in $L$ are visited within their time windows. If condition 4 is met, the vehicle is via path $p\left(L_{f}^{1}\right)$ always able to reach $v\left(L_{f}^{1}\right)$ before this time. For example by departing at 0 , the vehicle arrives at $v\left(L_{f}^{1}\right)$ at time $\delta_{L_{f}^{1}}(0)$ which is by condition 4 smaller than or equal to $\delta_{L_{f}^{2}}(0)$. Therefore, a departure at 0 over path $p\left(L_{f}^{1} \oplus L\right)$ does not violate any time windows. In conclusion, any extension $L$ of $p\left(L_{f}^{2}\right)$ to $2 n+1$ will be a feasible extension of $p\left(L_{f}^{1}\right)$ to $2 n+1$ as it results in an elementary path with does not violate time windows and capacity constraints.

Then for (ii), it still has to be proven that for all feasible extensions of $L$ of $p\left(L_{f}^{2}\right)$ to $2 n+1$ it holds that $\operatorname{obj}\left(L_{f}^{1} \oplus L\right) \geq$ $\operatorname{obj}\left(L_{f}^{2} \oplus L\right)$. Let $L_{f}^{1 *}=L_{f}^{1} \oplus L$ and $L_{f}^{2 *}=L_{f}^{2} \oplus L$. We also denote $t_{0}^{2}=$ $\operatorname{argmin}_{t \in \operatorname{dom}\left(L_{f}^{2 *}\right)}\left\{\delta_{L_{f}^{2 *}}(t)-t\right\}$ as the optimal departure time from the depot for path $p\left(L_{f}^{2 *}\right)$ and $r(L)$ as the sum of the profits associated with the nodes visited along path $p(L)$. The objective value of the path is:

$$
\begin{aligned}
\operatorname{obj}\left(L_{f}^{2 *}\right) & =r\left(L_{f}^{2 *}\right)-\left(\delta_{L_{f}^{2 *}}\left(t_{0}^{2}\right)-t_{0}^{2}\right) \\
& =r\left(L_{f}^{2}\right)+r(L)-\left(\delta_{L_{f}^{2 *}}\left(t_{0}^{2}\right)-t_{0}^{2}\right)
\end{aligned}
$$

Now consider the path $p\left(L_{f}^{1 *}\right)$ resulting from extending $L_{f}^{1}$ by $L$. Moreover, consider a departure time at the depot of this path of $t_{0}^{1}=\max \left\{0, t_{0}^{2}+\phi\left(L_{f}^{1}, L_{f}^{2}\right)\right\}$. The time $t_{0}^{1}$ is a feasible departure time for label $L_{f}^{1 *}$ because a departure time of 0 is always possible (as the extension of $L_{f}^{1}$ by $L$ is feasible) and by the definition of $\phi\left(L_{f}^{1}, L_{f}^{2}\right)$ in Eq. (35) $t_{0}^{2}+\phi\left(L_{f}^{1}, L_{f}^{2}\right)$ belongs to $\operatorname{dom}\left(L_{f}^{1}\right)$ if it is nonnegative. This departure time $t_{0}^{1}$ ensures that we reach node $v\left(L_{f}^{1}\right)$ at time $\delta_{L_{f}^{2}}\left(t_{0}^{2}\right)$ or earlier, meaning:

$\delta_{L_{f}^{1}}\left(t_{0}^{1}\right) \leq \delta_{L_{f}^{2}}\left(t_{0}^{2}\right)$. 
Moreover, as $t_{0}^{1}$ is a feasible departure time it can be used to compute a lower bound on $\operatorname{obj}\left(L_{f}^{1 *}\right)$ :

$$
\begin{aligned}
& \operatorname{obj}\left(L_{f}^{1 *}\right) \geq r\left(L_{f}^{1 *}\right)-\left(\delta_{L_{f}^{1 *}}\left(t_{0}^{1}\right)-t_{0}^{1}\right) \\
& \quad=r\left(L_{f}^{1}\right)+r(L)-\left(\delta_{L_{f}^{1 *}}\left(t_{0}^{1}\right)-\max \left\{0, t_{0}^{2}+\phi\left(L_{f}^{1}, L_{f}^{2}\right)\right\}\right) \\
& \geq r\left(L_{f}^{1}\right)+r(L)-\left(\delta_{L_{f}^{2 *}}\left(t_{0}^{2}\right)-\max \left\{0, t_{0}^{2}+\phi\left(L_{f}^{1}, L_{f}^{2}\right)\right\}\right) \\
& \geq r\left(L_{f}^{1}\right)+r(L)-\left(\delta_{L_{f}^{2 *}}\left(t_{0}^{2}\right)-t_{0}^{2}-\phi\left(L_{f}^{1}, L_{f}^{2}\right)\right) \\
& \geq r\left(L_{f}^{2}\right)-\phi\left(L_{f}^{1}, L_{f}^{2}\right)+r(L)-\left(\delta_{L_{f}^{2 *}}\left(t_{0}^{2}\right)-t_{0}^{2}-\phi\left(L_{f}^{1}, L_{f}^{2}\right)\right) \\
& \geq r\left(L_{f}^{2 *}\right)+r(L)-\left(\delta_{L_{f}^{2 *}}\left(t_{0}^{2}\right)-t_{0}^{2}\right)=\operatorname{obj}\left(L_{f}^{2 *}\right)
\end{aligned}
$$

Note that in inequality (39) we use the property derived at (37). Inequality (40) is derived by the simple fact that $\forall x \in$ $R:-\max \{0, x\} \leq-x$, and inequality (41) uses condition 5 of Proposition 4.1.

\subsection{The backward labeling algorithm}

In the backward labeling algorithm we start in the opposite direction and start generating labels from the end depot. The definition of a backward label is discussed in Section 4.2.1. The labels are extended as discussed in Section 4.2.2. The dominance criterion for backward labels is discussed in Section 4.2.3.

\subsubsection{Backward label}

In the backward labeling algorithm, labels are extended from the end depot $2 n+1$ to its predecessors. For a label $L_{b}$, we associate the following components:

$p\left(L_{f}\right) \quad$ The partial path of label $L_{b}$.

$v\left(L_{b}\right) * \quad$ The first node visited on the partial path $p\left(L_{b}\right)$.

$L^{-1}\left(L_{b}\right) *$ The parent label from which $L_{b}$ originates by extending it with $v\left(L_{b}\right)$.

$O\left(L_{b}\right) * \quad$ The set of incomplete requests, i.e., the delivery is visited but not the pickup node.

$U\left(L_{b}\right) * \quad$ The set of requests for which the delivery nodes are already visited along the partial path $p\left(L_{b}\right)$. It contains both the complete and incomplete requests. Therefore, $O\left(L_{b}\right) \subseteq U\left(L_{b}\right)$.

$P\left(L_{b}\right) \quad$ The set of pickup nodes of incomplete request in $p\left(L_{b}\right)$, i.e., $j \in N_{P}$ and $R_{j+n} \in O\left(L_{b}\right)$.

$D\left(L_{b}\right) \quad$ The set of delivery nodes not visited in $p\left(L_{b}\right)$, i.e., $j \in N_{D}$ and $R_{j-n} \notin U\left(L_{b}\right)$.

$q\left(L_{b}\right) * \quad$ The load of the tour after visiting node $v\left(L_{b}\right)$.

$\delta_{L_{b}}(t) * \quad$ The arrival time at the end node $2 n+1$ through the partial path represented by $L_{b}$ when leaving node $v\left(L_{b}\right)$ at time $t$.

$r\left(L_{b}\right)$ * The overall profits collected with the requests completed on the partial path $p\left(L_{b}\right)$.

Again, only the items marked with a ${ }^{*}$ are stored in the label and the sets $D\left(L_{b}\right)$ and $P\left(L_{b}\right)$ can be deduced from the sets $O\left(L_{b}\right)$ and $U\left(L_{b}\right)$. Furthermore, the partial path can be deduced from iteratively checking the first node visited in the parent label of which the this label was an extension.

\subsubsection{Label extension}

Let $\operatorname{dom}\left(L_{b}\right)$ be the domain of the function $\delta_{L_{b}}(t)$ and let $t_{l}\left(L_{b}\right)$ denote the latest possible ready time at $v\left(L_{b}\right): t_{l}\left(L_{b}\right)=$ $\max \left(\operatorname{dom}\left(L_{b}\right)\right)$. We extend a label $L_{b}^{\prime}$ along an $\operatorname{arc}\left(j, v\left(L_{b}^{\prime}\right)\right)$ to create a new label $L_{b}$. To be a feasible extensions at least the following two conditions should be met:

$t_{l}\left(L_{b}\right) \geq \max \left\{t_{m}, e_{j}+s_{j}\right\} \quad \wedge \quad j \in N \backslash\{2 n+1\}$

$q\left(L_{b}\right)+q_{j} \leq Q \quad \wedge \quad j \in N \backslash\{2 n+1\}$

Condition (43) ensures that node $j$ can be reached within its time window and that the extension will be stopped before $t_{m}$ is exceeded, while condition (44) ensures capacity feasibility. Furthermore, $L_{b}^{\prime}$ and $j$ must satisfy one of the following three conditions:

$j+n \in O\left(L_{b}^{\prime}\right) \wedge j \in N_{P}$

$j \notin U\left(L_{b}^{\prime}\right) \wedge j \in N_{D}$

$O\left(L_{f}^{\prime}\right)=\emptyset \wedge j=0$

Condition (45) indicates that if $j$ is a pickup node, the corresponding delivery node should have been visited already. Furthermore, condition (46) states that if $j$ is a delivery node, it should not have been visited before. Finally, condition (47), states that if $j$ is the begin depot then all visited requests should have been completed. In the presence of those conditions, only elementary paths that satisfy precedence constraint (11) are generated.

At last, it needs to be checked that all pickup nodes of incomplete requests for which the delivery node is included in $p\left(L_{b}^{\prime}\right)$ can be visited before node $j$. To do so, first the latest possible arrival time at node $j$ which ensures a ready time of $t_{l}\left(L_{b}\right)$ at $v\left(L_{b}\right)$ should be determined. Let this be denoted by $t_{r}\left(j, L_{b}^{\prime}\right)$, then it holds that $t_{r}\left(j, L_{b}^{\prime}\right)=\min \left\{l_{j}, \max \left\{t: t+s_{j}+\tau_{j v\left(L_{b}^{\prime}\right)}(t)+s_{v\left(L_{b}^{\prime}\right)} \leq t_{l}\left(L_{b}^{\prime}\right)\right\}\right.$. As shown $t_{r}\left(j, L_{b}^{\prime}\right)$ is determined by the latest possible arrival time at $j$ (i.e., $l_{j}$ ) or by the latest possibility departure time to reach $v\left(L_{b}^{\prime}\right)$ on time.

Then, all nodes from which the vehicle cannot depart before time $t_{r}\left(j, L_{b}^{\prime}\right)$ due to time window constraints, cannot be visited before node $j$ and are defined as unreachable. This can be made stronger by considering the travel time to node $j$ as well. However, again due to the absence of the triangle inequality and the time dependent travel times we cannot simply consider the travel time of the direct connection. Therefore, we need for any unvisited node $k$ the latest possible departure time to arrive at $j$ at time $t_{r}\left(j, L_{b}^{\prime}\right)$, which can be computed by $\left.\max \left\{t: t+\tau_{k j}(t) \leq t_{r}\left(j, L_{b}^{\prime}\right)\right)\right\}$. Finally, the latest possible time the vehicle could depart from any node before node $j$ is given by $t_{d}\left(j, L_{f}^{\prime}\right)=\max _{k \in P\left(L_{f}^{\prime}\right) \cup D\left(L_{f}^{\prime}\right)}\{\max \{t$ : $\left.\left.t+\tau_{k j}(t) \leq t_{r}\left(j, L_{b}^{\prime}\right)\right\}\right\}$.

This means that any node $k$ with the earliest allowed arrival time $e_{k}$ later than this time (i.e., $l_{k}>t_{d}\left(j, L_{f}^{\prime}\right)$ ) cannot be a predecessor of node $j$, also not in an indirect way as no node could be left after $t_{d}\left(j, L_{f}^{\prime}\right)$ and still reaching node $j$ on time.

If a pickup node $k$ cannot be a predecessor of node $j$ and its corresponding delivery node is already visited (i.e., $k+n \in O\left(L_{f}^{\prime}\right)$ ), the extension to $j$ is not feasible as the item which should be delivered cannot be picked up anymore. Therefore, as stated in condition (48), all pickup nodes of requests of which the delivery node is already visited in $p\left(L_{b}^{\prime}\right)$ should be a possible predecessor of $j$ to make sure that extending label $L_{b}^{\prime}$ to $j$ is feasible.

$l_{k} \leq t_{d}\left(j, L_{b}^{\prime}\right) \quad \forall k \in P\left(L_{b}^{\prime}\right): k \neq j$ 
(a)

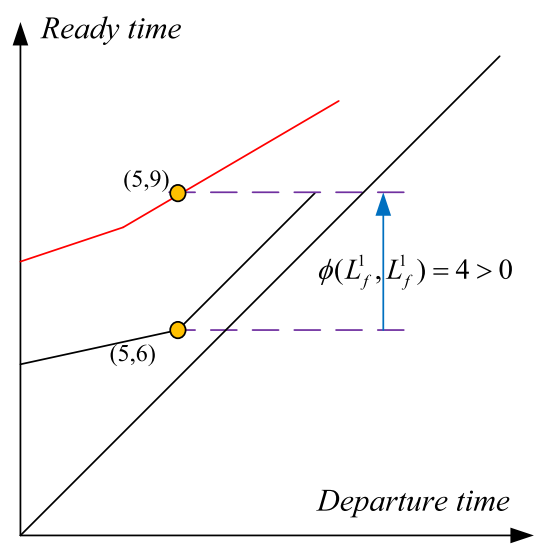

Ready time for label $L_{f}^{1}$ (b)

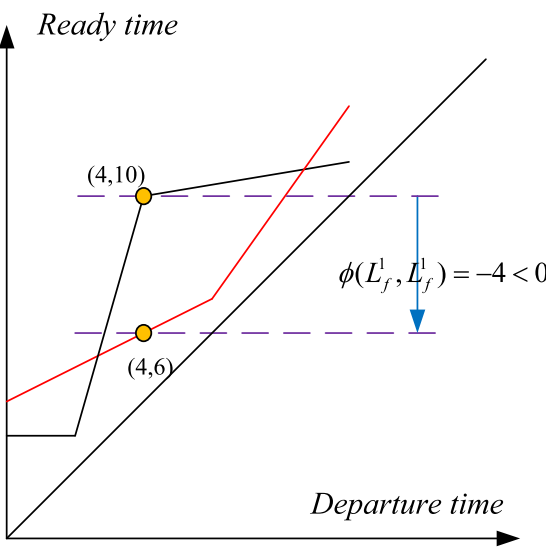

Ready time for label $L_{f}^{2}$

Fig. 4. Illustration of $\phi\left(L_{f}^{1}, L_{f}^{2}\right)$.

If the extension along the $\operatorname{arc}\left(j, L_{b}^{\prime}\right)$ is feasible according to the provided conditions, the information in label $L_{b}$ is set as follows:

$L^{-1}\left(L_{b}\right)=L_{b}^{\prime}$

$v\left(L_{b}\right)=j$

$\delta_{L_{b}}(t)=\delta_{L_{b}^{\prime}}\left(\max \left\{e_{v\left(L_{b}^{\prime}\right)}, t+\tau_{j v\left(L_{b}^{\prime}\right)}(t)\right\}+s_{v\left(L_{b}^{\prime}\right)}\right)$

$q\left(L_{b}\right)=q\left(L_{b}^{\prime}\right)+q_{j}$

$r\left(L_{b}\right)= \begin{cases}r\left(L_{b}^{\prime}\right)+r_{j} & \text { if } j \in N_{P}, \\ r\left(L_{b}^{\prime}\right) & \text { otherwise. }\end{cases}$

$O\left(L_{b}\right)= \begin{cases}O\left(L_{b}^{\prime}\right) \backslash\{j\} & \text { if } j \in N_{P}, \\ O\left(L_{b}^{\prime}\right) \cup\{j-n\} & \text { if } j \in N_{D}\end{cases}$

$U\left(L_{b}\right)= \begin{cases}U\left(L_{b}^{\prime}\right) \cup\{j-n\} & \text { if } j \in N_{D}, \\ U\left(L_{b}^{\prime}\right) & \text { otherwise. }\end{cases}$

\subsubsection{Label dominance}

Dominance of the backward algorithm can be constructed in the same way as in the case of the forward algorithm, because the arrival time functions are non-decreasing and stepwise linear as before.

Similar to the forward algorithm, in Proposition 4.2, we extend the set $U\left(L_{b}\right)$ to $\widetilde{U}\left(L_{b}\right)$ by including request of which the pickup or delivery node cannot be a predecessor of $v\left(L_{b}\right)$. Via the same reasoning as in Section 4.2.2 it can be derived that the latest possible time a vehicle could depart from a node to reach $v\left(L_{b}\right)$ on time is $t_{d}\left(L_{b}\right)=\max _{j \in P\left(L_{b}\right) \cup D\left(L_{b}\right)}\left\{\max \left\{t: t+\tau_{j v\left(L_{b}\right)}(t) \leq t_{l}\left(L_{b}\right)-\right.\right.$ $\left.s_{v\left(L_{b}\right)}\right\}$. This means that any node $j$ with the earliest possible departure time $e_{j}+s_{j}$ later than this time (i.e., $\left.e_{j}+s_{j}>t_{d}\left(L_{b}\right)\right)$ cannot be a predecessor of $v\left(L_{b}\right)$. Therefore, the corresponding request cannot be served anymore and can be added to the set $\widetilde{U}\left(L_{b}\right)$.

Furthermore, we define $\phi\left(L_{b}^{1}, L_{b}^{2}\right)$ (see Fig. 4 ) as:

$\phi\left(L_{b}^{1}, L_{b}^{2}\right)=\max \left\{x \in R: \delta_{L_{b}^{1}}(t)+x \leq \delta_{L_{b}^{2}}(t), \quad \forall t \in \operatorname{dom}\left(L_{b}^{2}\right)\right\}$

Then the dominance criterion will become:

Proposition 4.2. Label $L_{b}^{2}$ is dominated by label $L_{b}^{1}$ if

1. $v\left(L_{b}^{1}\right)=v\left(L_{b}^{2}\right)$

2. $U\left(L_{b}^{1}\right) \subseteq \widetilde{U}\left(L_{b}^{2}\right)$
3. $O\left(L_{b}^{1}\right)=O\left(L_{b}^{2}\right)$

4. $t\left(L_{b}^{1}\right) \geq t\left(L_{b}^{2}\right)$

5. $r\left(L_{b}^{1}\right) \geq r\left(L_{b}^{2}\right)-\phi\left(L_{b}^{1}, L_{b}^{2}\right)$

6. $q\left(L_{b}^{1}\right) \leq q\left(L_{b}^{2}\right)$

For the proof of Proposition 4.2 the same reasoning as the proof of Proposition 4.1 could be followed.

\subsection{Merging forward and backward labels}

When all forward and backward labels are generated, they are merged to construct feasible profitable tours. A forward label $L_{f}$ and a backward label $L_{b}$ can be merged if the following conditions are satisfied:

1. $v\left(L_{f}\right)=v\left(L_{b}\right)$

2. $O\left(L_{f}\right) \cap O\left(L_{b}\right)=\left\{v\left(L_{f}\right)\right\}$

3. $\left(U\left(L_{f}\right) \backslash O\left(L_{f}\right)\right) \cap\left(U\left(L_{b}\right) \backslash O\left(L_{b}\right)\right)=\emptyset$

4. $q\left(L_{f}\right)+q\left(L_{b}\right)=q_{v\left(L_{f}\right)}$

5. $\operatorname{Img}\left(L_{f}\right) \cap \operatorname{dom}\left(L_{b}\right) \neq \emptyset$

The resulting path $p(L)=\left(p\left(L_{f}\right) \oplus p\left(L_{b}\right)\right)$ has the following attributes:

1. $v(L)=2 n+1$

2. $r(L)=r\left(L_{f}\right)+r\left(L_{b}\right)-r_{v\left(L_{f}\right)}$

3. $O(L)=\emptyset$

4. $U(L)=U\left(L_{f}\right) \cup U\left(L_{b}\right)$

5. $q(L)=0$

6. $\delta_{L}(t)=\delta_{L_{b}}\left(\delta_{L_{f}}(t)\right), \forall t \in \operatorname{dom}\left(L_{f}\right)$ such that $\delta_{L_{f}}(t) \in \operatorname{dom}\left(L_{b}\right)$

However, this bidirectional labeling algorithm can generate duplicate solutions. Consider a feasible solution $p^{*}$ including nodes $i$, $j$ and $k$ in this order. Each node $x \in p^{*}$ is associated with a forward label $L_{f}(x)$ and a backward label $L_{b}(x)$ (i.e., $\left.v\left(L_{f}(x)\right)=v\left(L_{b}(x)\right)=x\right)$. Therefore, the path $p^{*}$ can be obtained by merging $L_{f}(i)$ with $L_{b}(i)$ as well as merging by $L_{f}(j)$ with $L_{b}(j)$. To overcome this drawback, we devised an additional test: we accept a solution only when a further extension of the forward label is impossible. In our example (see Fig. 5) the extension from $L_{f}(i)$ to node $j$ is feasible and the extension from $L_{f}(j)$ to node $k$ is infeasible by the predefined fixed time $t_{m}$. We generate solution $p^{*}$ by merging $L_{f}(j)$ and $L_{b}(j)$ instead of $L_{f}(i)$ and $L_{b}(i)$. The test is performed for each candidate pair of labels and guarantees that each path is generated only once. 


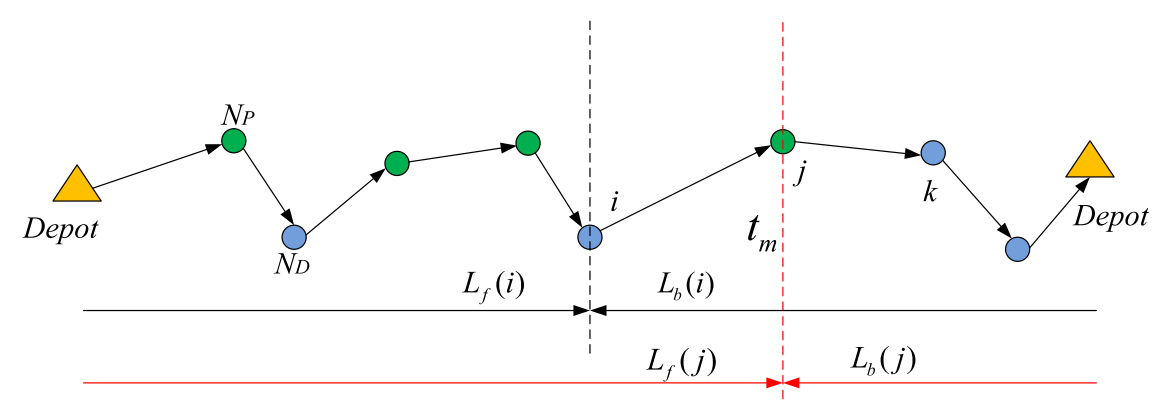

Fig. 5. An illustration of preventing duplicate solutions.

\section{Restricted dynamic programing heuristic algorithm}

Although the tailored labeling algorithm returns the optimal solution of our proposed problem, it is not fast enough to solve problems of realistic size within reasonable computation time.

In order to avoid enormous computation times and to save memory usage, Malandraki and Dial (1996) proposed a restricted dynamic programing heuristic algorithm for the TSP. The main concept is to limit in each stage the number of partial paths for further extension by a given parameter $B$. Furthermore, in each stage all partial paths should have the same number of visited nodes. Malandraki and Dial (1996) show that increasing the value of $B$ results in better solutions, but also in substantial higher computation times. Note that $B=1$ results in a nearest neighborhood heuristic and $B=\infty$ results it an exact dynamic programing algorithm.

Gromicho, van Hoorn, Kok, and Schutten (2012) propose a restricted dynamic programing algorithm for solving realistic VRPs and restrict the state space even further, by using a form of beam search (Bisiani, 1987), which means that each partial path is only expanded to $E$ of its nearest feasible nodes.

The same principle of restricting the number of extension of a partial path can be applied to our labeling algorithm as well with some minor changes. Because of the precedence constraints, expanding a partial path to a pickup node may improve or deteriorate the objective function value depending on the profit of that node and the additional travel time to visit that node. However, expanding to a delivery node can only decrease the objective function value as additional travel time is necessary to visit that node while no profit is assigned the node. Moreover, because of the time-dependent travel time, the objective function value of a partial path is determined by its optimal departure time at the origin depot, which might change after a certain extension.

Therefore, it is not sufficient to select the partial paths with the highest objective function value, and we introduce a new selection method for each stage taking global information into account. For every extension of a partial path the $E / 2$ best expanded partial paths ending with a pickup node and the $E / 2$ best expanded partial paths ending with a delivery node are selected. Furthermore, in each stage we select the best $B / 2$ partial paths that expand to a pickup node and the best $B / 2$ partial paths that expand to a delivery node for further expansion. Moreover, instead of just using the objective function value for the selection, we use the earliest arrival time to order the expanded partial paths. If two expanded partial paths have the same earliest arrival time, the objective function value will be used to order them.

The restricted dynamic programing heuristic is described in Algorithm 1 . Herein, an empty labels set $Z_{k}$ is created for each stage $k$ for further extension (Step 2). Then the first label which represents the start from the depot is generated and put into the set $Z_{0}$ (Steps 3 and 4 ). If the number of the labels stored in each stage $k$ is larger than $B$, only the $B$ best labels are selected for

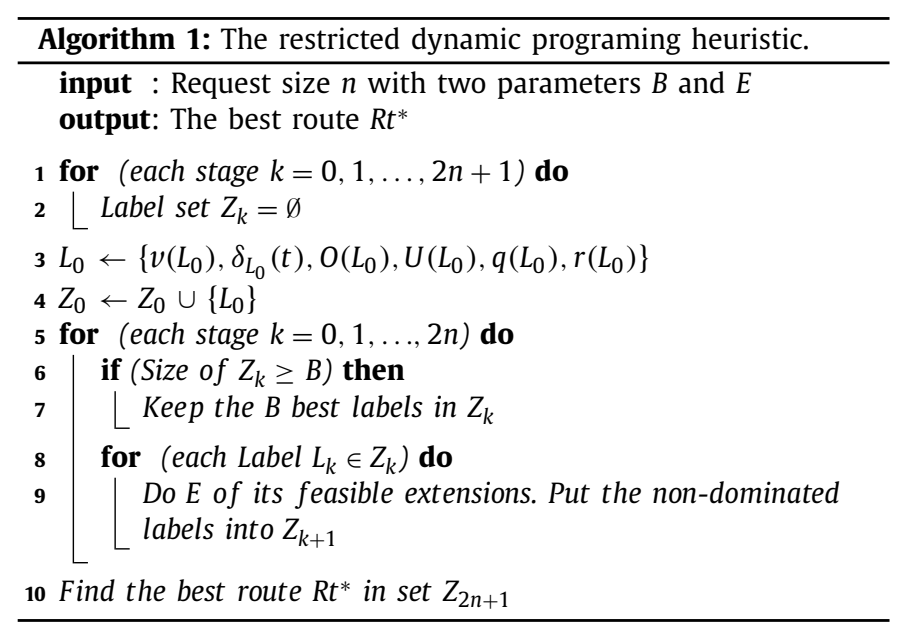

Table 2

Characteristics of the TDPPDPTW and its variants instances.

\begin{tabular}{lll}
\hline Group & $Q$ & $W$ \\
\hline AA & 15 & 60 \\
BB & 20 & 60 \\
CC & 15 & 120 \\
DD & 20 & 120 \\
\hline
\end{tabular}

further extension (Step 7). Moreover, for each label in stage $k$, only $E$ of its feasible extensions are made and put in $Z_{k+1}$ (Step 9). In the end, the best route $R t^{*}$ will be found by checking the labels in $Z_{2 n+1}$ (Step 10).

Note that there is no optimality guarantee anymore with such a restricted dynamic programing heuristic algorithm.

\section{Computational results}

In this section the computational experiments are discussed. The algorithms are coded in JAVA and all computations are carried out on a single thread of a server with four CPU's (2.4 gigahertz/6 cores) and 64 gigabytes RAM. Each run has a time limit of 2 weeks and a maximum memory allowance of 16 gigabytes RAM. For our numerical study we use an adapted version of the instances for the PDPTW in Ropke etal. (2009). In these instances, the coordinates of each pickup and delivery location are both randomly and uniformly distributed over a $[0,50] \times[0,50]$ square and a single depot is located in this square. The load $q_{i}$ for each request $R_{i}$ is also randomly selected from the interval $[5, Q]$, where $Q$ is the vehicle capacity. Table 2 summarizes the characteristic of these instances including the vehicle capacity $Q$ and the width of the time windows $W$. For each group, there are 10 instances with the number 


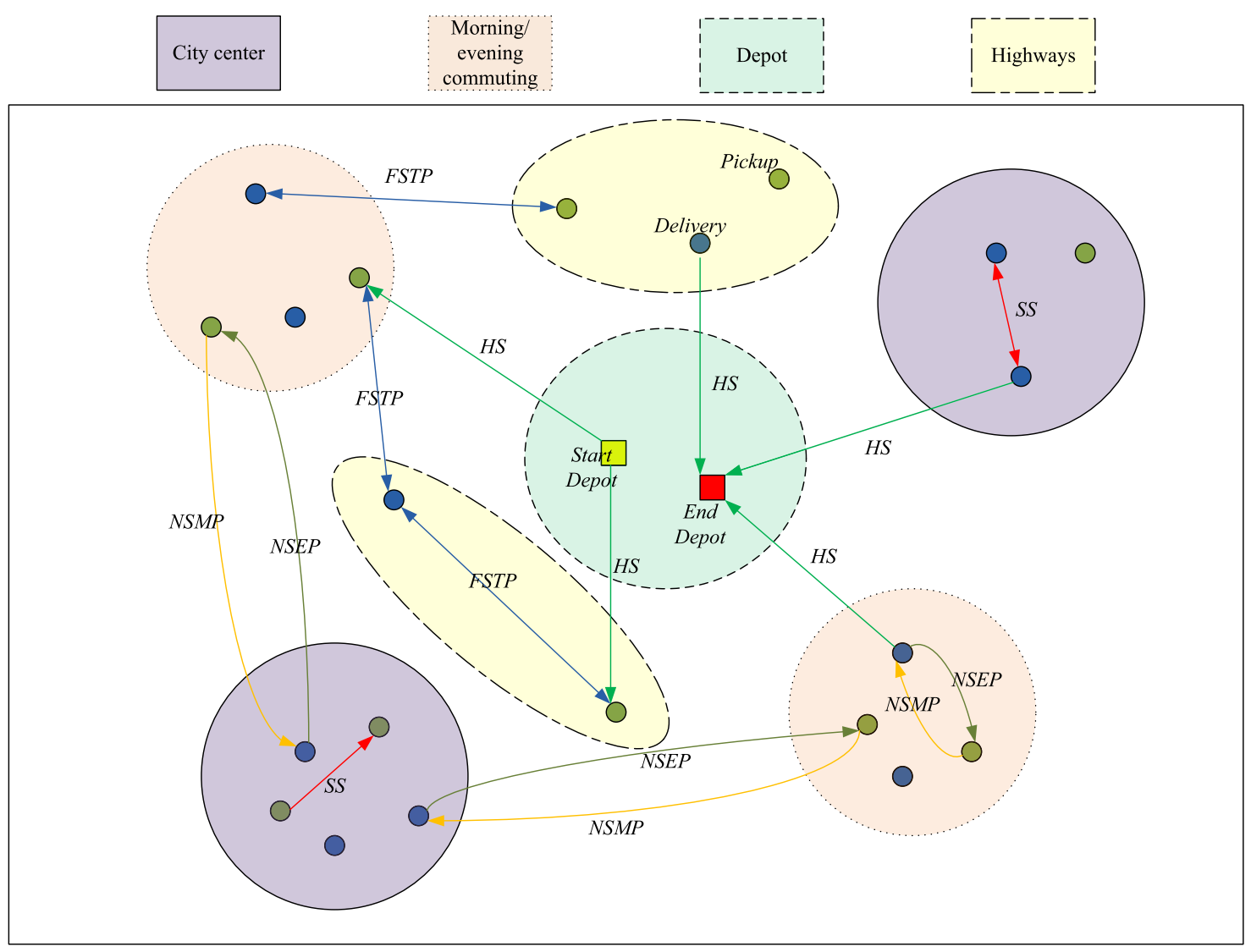

Fig. 6. An illustration of a instance with different speed profiles.

of requests ranging from 30 to 75 . For more details, we refer to Ropke et al. (2009).

In addition to the instances proposed by Ropke et al. (2009), we created for each class four new small instances with the amount of requests ranging from 10 to 25 . They are generated by only keeping the first $\alpha$ requests of the instance with 30 requests of each class, where $\alpha=10,15,20$ and 25. For example, the instance AA10 is created by considering the first ten requests of AA30.

In our instances, the coordinates, time windows and loads of the depot, pickup nodes and delivery nodes are the same as in the instances of Ropke etal. (2009). Furthermore, the vehicle capacity $Q$ is also the same. In the instances of Ropke et al. (2009) the travel time is fixed and no profits were assigned to the requests.

To make the travel time time-dependent, road congestion is handled by a so-called speed model which consists of different speed profiles. It is used to determine the travel time between two nodes on a specific departure time. This speed model is based on the speed model of Verbeeck etal. (2014) for the TDOP and Dabia et al. (2013) for the TDVRPTW. Without loss of generality, we assume that breakpoints are the same for all speed profiles as congestion tends to happen around the same time regardless of the type of speed profile. The pickup and delivery node of each request is randomly assigned to one of the three predefined areas: morning and evening commuting area, city center and highways. Then, the speed profile of a link is assigned according to the type (e.g., depot or request node) and location of the tail node and head node.

In our speed model, the planning horizon covers 14 working hours (840 minutes, from 7 a.m. to 9 p.m.) and a minute is set to be one unit of time, while, in Ropke etal. (2009), the planning horizon of length 600 minutes is considered. Each speed profile has four non-overlapping time periods with constant speed, reflecting two
Table 3

Speed profiles.

\begin{tabular}{lllll}
\hline $\begin{array}{l}\text { Congestion } \\
\text { description }\end{array}$ & $\begin{array}{l}\text { Morning } \\
\text { peak }\end{array}$ & Normal & $\begin{array}{l}\text { Evening } \\
\text { peak }\end{array}$ & Normal \\
Time periods & 7 a.m.-9 a.m. & 9 a.m.-5 p.m. & 5 p.m.-7 p.m. & 7 p.m.-9 p.m. \\
\hline 1. SS & 0.5 & 0.81 & 0.5 & 0.81 \\
2. NSMP & 0.67 & 1.33 & 0.88 & 1.33 \\
3. NSEP & 0.88 & 1.33 & 0.67 & 1.33 \\
4. FSTP & 0.85 & 1.5 & 0.85 & 1.5 \\
5. HS & 1.0 & 2.0 & 1.0 & 2.0 \\
\hline
\end{tabular}

congested periods and two periods with normal traffic conditions. Five speed profiles are included (see Fig. 6 and Table 3):

- Slow speed (SS): these links represent a busy central business district (CBD) with a lot of traffic during the whole day.

- Normal speed with morning peak (NSMP): these links represent roads leading from a residential area to the $\mathrm{CBD}$. These roads are in most cases congested in the morning.

- Normal speed with evening peak (NSEP): these links represent roads leading from a CBD to a residential zone. The roads typically encounter evening congestion.

- Fast speed with two peaks (FSTP): these links represent roads near the highway with a morning and evening peak in both directions.

- High speed (HS): these links connect the request nodes with the depot .

As in Ropke etal. (2009) all requests needed to be served no profits were assigned to the requests. To come up with meaningful settings for the profits we did preliminary tests with 20,40 and 80 units of profit assigned to each pickup node. As shown in Table 4, if the profit of the requests is 20 , it will be too low. There are even 
Table 4

Comparison of results for different profits settings.

\begin{tabular}{|c|c|c|c|c|c|c|c|c|c|}
\hline \multirow[b]{2}{*}{ Instance } & \multicolumn{3}{|c|}{20 units profit for each request } & \multicolumn{3}{|c|}{40 units profit for each request } & \multicolumn{3}{|c|}{80 units profit for each request } \\
\hline & $\begin{array}{l}\text { Optimal } \\
\text { value }\end{array}$ & $\begin{array}{l}\text { Profits } \\
\text { (served) }\end{array}$ & $\begin{array}{l}\text { Traveling } \\
\text { cost }\end{array}$ & $\begin{array}{l}\text { Optimal } \\
\text { value }\end{array}$ & $\begin{array}{l}\text { Profits } \\
\text { (served) }\end{array}$ & $\begin{array}{l}\text { Traveling } \\
\text { cost }\end{array}$ & $\begin{array}{l}\text { Optimal } \\
\text { value }\end{array}$ & $\begin{array}{l}\text { Profits } \\
\text { (served) }\end{array}$ & $\begin{array}{l}\text { Traveling } \\
\text { cost }\end{array}$ \\
\hline AA10 & 0 & $0(0)$ & 0 & 27.14 & $80(2)$ & 52.86 & 196.22 & $400(5)$ & 203.78 \\
\hline AA15 & 0 & $0(0)$ & 0 & 37.77 & $120(3)$ & 82.23 & 392.37 & $880(11)$ & 487.63 \\
\hline AA20 & 0 & $0(0)$ & 0 & 73.7 & $240(6)$ & 166.3 & 566.03 & $1200(15)$ & 633.97 \\
\hline AA25 & 9.06 & $60(3)$ & 50.94 & 203.01 & $560(14)$ & 356.99 & 884.41 & $1440(18)$ & 555.59 \\
\hline AA30 & 13.07 & $60(3)$ & 46.93 & 266.72 & $640(16)$ & 373.28 & 1020.86 & $1600(20)$ & 579.14 \\
\hline BB10 & 0 & $0(0)$ & 0 & 39.26 & $120(3)$ & 80.74 & 247.65 & $720(9)$ & 472.35 \\
\hline BB15 & 6.61 & $20(1)$ & 13.39 & 99.58 & $280(7)$ & 180.42 & 518.03 & $960(12)$ & 441.97 \\
\hline BB20 & 6.61 & $20(1)$ & 13.39 & 138.05 & $600(15)$ & 461.95 & 738.05 & $1200(15)$ & 461.95 \\
\hline BB25 & 6.25 & $40(2)$ & 33.75 & 147.88 & $520(13)$ & 372.12 & 787.64 & 1360 (17) & 572.36 \\
\hline BB30 & 4.26 & $20(1)$ & 15.74 & 274.45 & $840(21)$ & 565.55 & 1114.45 & $1680(21)$ & 565.55 \\
\hline CC10 & 2.81 & $40(2)$ & 37.19 & 57.63 & $160(4)$ & 102.37 & 340.2 & $560(7)$ & 219.8 \\
\hline CC15 & 5.69 & $40(2)$ & 34.31 & 98.29 & $280(7)$ & 181.71 & 469.59 & $960(12)$ & 490.41 \\
\hline CC20 & 4.35 & $20(1)$ & 15.65 & 97.27 & $480(12)$ & 382.73 & 712.53 & $1280(16)$ & 567.47 \\
\hline CC25 & 9.42 & $60(3)$ & 50.58 & 226.23 & 720 (18) & 493.77 & 984.3 & $1600(20)$ & 615.7 \\
\hline CC30 & 3.73 & $60(3)$ & 56.27 & 316.39 & $800(20)$ & 483.61 & 1167.47 & $1760(22)$ & 592.53 \\
\hline DD10 & 11.8 & $60(3)$ & 48.2 & 85.88 & $160(4)$ & 74.12 & 315.34 & 720 (9) & 404.66 \\
\hline DD15 & 10.18 & $60(3)$ & 49.82 & 99.05 & $200(5)$ & 100.95 & 554.55 & 1040 (13) & 485.45 \\
\hline DD20 & 14.44 & $80(4)$ & 65.56 & 182.14 & $640(16)$ & 457.86 & 832.05 & 1360 (17) & 527.95 \\
\hline DD25 & 6.66 & $80(4)$ & 73.34 & 250.16 & $680(17)$ & 429.84 & 1025.75 & $1600(20)$ & 574.25 \\
\hline DD30 & 16.9 & $80(4)$ & 63.1 & 343.65 & $840(21)$ & 496.35 & 1247.22 & $1840(23)$ & 592.78 \\
\hline
\end{tabular}

Table 5

Comparison of the tailored labeling algorithm without and with dominance and feasibility check.

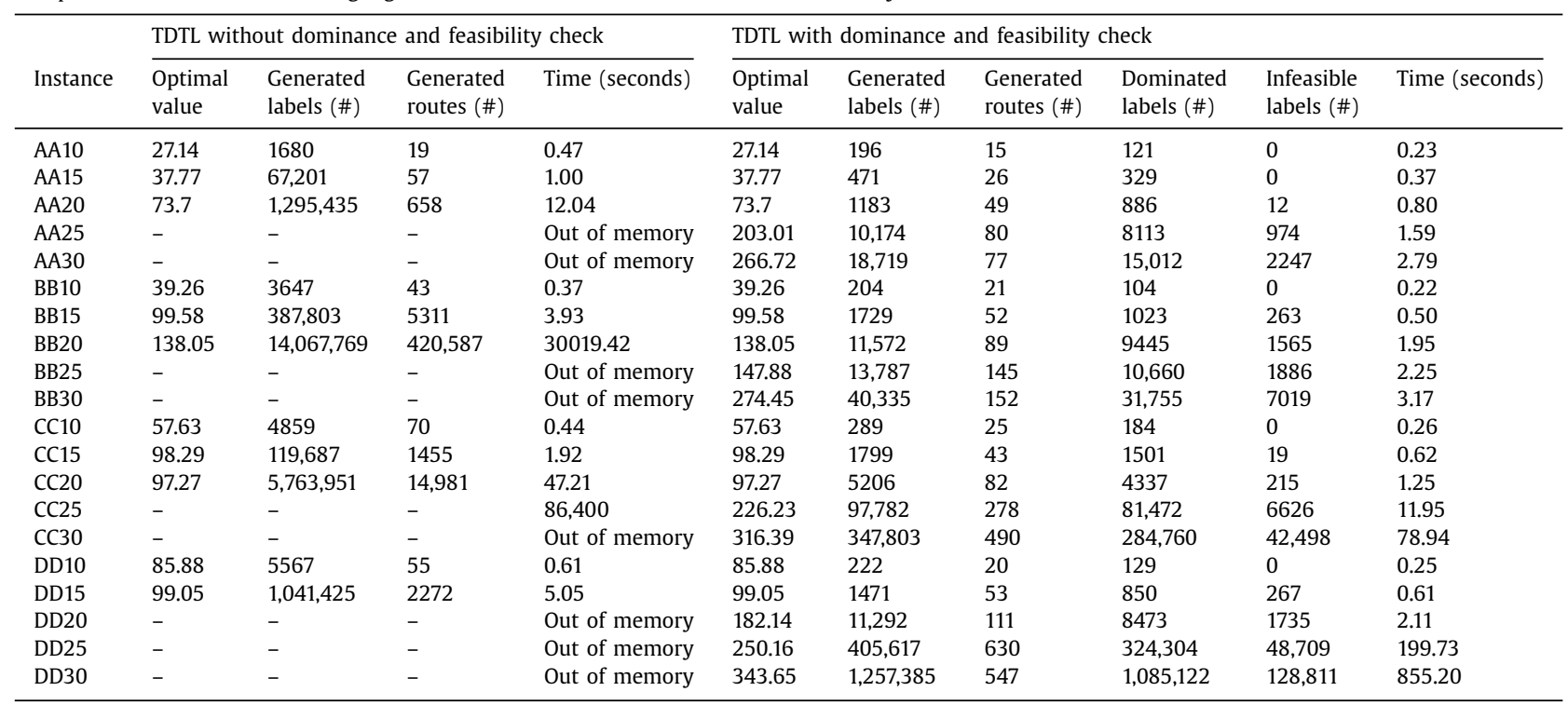

routes in which it is best to do nothing. If the profit per request is 40 , on average $56 \%$ of the requests are served and the total profit in the route is $52 \%$ higher than the traveling cost. When the profits increase to 80 , the provider would like to serve more request (on average $74 \%$ ) and the generated profits are way (on average 141\%) higher than the traveling cost. To avoid bias on either profits or traveling cost, in the remainder of the computational experiments, we decided to assign a profit of 40 units to all pickup nodes, which makes a request only profitable if the additional travel time to serve the request (i.e., visiting both the pickup and delivery node) is less than 40 time units.

\subsection{Performance of the tailored labeling algorithm}

Table 5 gives the results of the experiments using the tailored labeling algorithm without dominance and feasibility check and the tailored labeling algorithm with dominance and feasibility check. In Table 5, we observe that the tailored labeling algorithm without dominance and feasibility check can only solve 11 instances (out of 20 instances) to optimality within the given memory limit (16 gigabytes) or time limit ( 1 day). In contrast, the tailored labeling algorithm with dominance and feasibility check is able to solve all of the instances within a reasonable running time (within 15 minutes). Moreover, due to the dominance and feasibility check much less labels and routes (columns 7 and 8) are generated compared to its counterpart (columns 3 and 4). Therefore, it shows that the proposed dominance criterion and feasibility check both show their great potentials to get rid of the unpromising labels (columns 9 and 10) which are proven not to be the part of the optimal solution. On average $84 \%$ of the labels are dominated and about $11 \%$ is proven to lead to an infeasible extension.

In Table 6 , we present the results of the experiments using the mathematical model introduced in Section 3.2, solved by the optimization software Gurobi (version 5.6) with its default parame- 
Table 6

Results of solving the mathematical model by using the Gurobi.

\begin{tabular}{llllll}
\hline Instances & LB & Best & Time (seconds) & Gap (\%) & Gap_opt (\%) \\
\hline AA10 & 27.14 & 27.14 & 1.95 & 0 & 0 \\
AA15 & 37.77 & 37.77 & 4.66 & 0 & 0 \\
AA20 & 73.7 & 73.7 & 24.93 & 0 & 0 \\
AA25 & 203.01 & 203.01 & 46344.06 & 0 & 0 \\
AA30 & 216.34 & 543.47 & 86,400 & 151 & 50.92 \\
BB10 & 39.26 & 39.26 & 1.451 & 0 & 0 \\
BB15 & 99.58 & 99.58 & 121.03 & 0 & 0 \\
BB20 & 138.05 & 138.05 & 1669.74 & 0 & 0 \\
BB25 & 147.88 & 147.88 & 48616.51 & 0 & 0 \\
BB30 & 245.7 & 515.404 & 86,400 & 110 & 46.75 \\
CC10 & 57.63 & 57.63 & 3.29 & 0 & 0 \\
CC15 & 98.29 & 98.29 & 44.34 & 0 & 0 \\
CC20 & 97.27 & 97.27 & 7935.29 & 0 & 0 \\
CC25 & 209.08 & 1316.69 & 86,400 & 530 & 82.82 \\
CC30 & 237.02 & 1674.74 & 86,400 & 607 & 81.11 \\
DD10 & 85.88 & 85.88 & 1.43 & 0 & 0 \\
DD15 & 99.05 & 99.05 & 54.65 & 0 & 0 \\
DD20 & 182.14 & 182.14 & 4959.48 & 0 & 0 \\
DD25 & 221.39 & 1419.81 & 86,400 & 541 & 82.38 \\
DD30 & 279.51 & 1733.62 & 86,400 & 520 & 80.18 \\
\hline
\end{tabular}

ter settings and a computation time limit of 1 day. For each instance, we provide the lower bound (LB), best feasible solution found (Best), the processing time given in seconds (Time) and the gap (Gap) provided by Gurobi and the relative gap with respect to the optimal solution (Gapopt). The relative gap is calculate by Gap $_{\text {opt }}=100 \times \frac{\text { Best }-O p t}{\text { Best }}$ in which Opt is the value of the optimal solution for the instance derived by our tailored labeling algorithm. As shown in Table 6, the proposed mathematical model is able to solve instances with up to 25 requests within the time limit. However, for larger instances, it takes already more than one day compared to the at maximum 15 minutes of our tailored labeling algorithm.

\subsection{Results of the tailored labeling algorithm on all instances}

In Table 7, we report the results of our tailored labelling algorithm. We present the computation times for both the bidirectional search (i.e., $t_{m}=420$, the middle of the time horizon) and the mono-directional search (i.e., $t_{m}=840$, the end of the time horizon). Since both algorithms lead to the optimal value, this value is presented only once (column 2). The bidirectional search shows greater potential power in terms of computation time. For some instances (DD40 and DD50), the bidirectional search is even 10 times faster than the monodirectional search. Moreover, the bidirectional search is capable of finding the optimal value of more instances than the monodirectional search within our set computation time limit of 2 weeks. This is mainly because of the fact that the number of labels needed to be processed in the bidirectional search is considerably less compared to the mono-directional search. However, merging the forward and backward labels also requires some computational effort. It can be seen that for 6 easy instances (AA35, BB30, BB40, BB50, BB55, BB70) the extra time necessary to merge the labels outweighs the reduced time to generate labels in the bidirectional search. As we prefer an approach which is also able to solve difficult instances we focus in the remainder of this paper at the bidirectional search.

With the bidirectional search, 34 of the 40 instances could be solved within our time limit of 2 weeks. We did not find optimal solutions for the instances with more than 65 requests in the CC group and more than 55 requests in the DD group. This means that the algorithm was still generating labels after 2 weeks, and therefore also no upper bound is available.

It is not a surprise that the computation time increases with the number of requests. However, Table 7 also demonstrates that the
Table 7

Monodirectional algorithm vs. bidirectional algorithm.

\begin{tabular}{|c|c|c|c|c|}
\hline \multirow[b]{2}{*}{ Instance } & \multirow[b]{2}{*}{$\begin{array}{l}\text { Optimal } \\
\text { value }\end{array}$} & \multirow[b]{2}{*}{$\begin{array}{l}\text { Requests } \\
\text { served }\end{array}$} & \multicolumn{2}{|c|}{ Processing time (seconds) } \\
\hline & & & Monodirectional & Bidirectional \\
\hline AA30 & 266.72 & 16 & 2.37 & 2.12 \\
\hline AA35 & 278.14 & 20 & 4.68 & 5.04 \\
\hline AA40 & 341.74 & 22 & 7.94 & 5.82 \\
\hline AA45 & 361.57 & 21 & 23.54 & 5.6 \\
\hline AA50 & 430.41 & 23 & 22.40 & 9.53 \\
\hline AA55 & 436.05 & 27 & 32.79 & 18.66 \\
\hline AA60 & 505.02 & 26 & 87.11 & 24.71 \\
\hline AA65 & 576.39 & 29 & 182.09 & 34.80 \\
\hline AA70 & 606.68 & 29 & 180.01 & 39.14 \\
\hline AA75 & 715.53 & 31 & 1038.67 & 257.60 \\
\hline BB30 & 274.45 & 21 & 2.36 & 2.78 \\
\hline BB35 & 337.55 & 22 & 1.30 & 1.03 \\
\hline BB40 & 326.82 & 23 & 8.30 & 9.95 \\
\hline BB45 & 378.90 & 23 & 34.88 & 27.94 \\
\hline BB50 & 432.10 & 25 & 31.81 & 37.41 \\
\hline BB55 & 530.84 & 27 & 40.14 & 59.02 \\
\hline BB60 & 558.02 & 28 & 164.38 & 119.2 \\
\hline BB65 & 547.84 & 25 & 353.15 & 332.13 \\
\hline BB70 & 558.29 & 27 & 225.79 & 351.57 \\
\hline BB75 & 613.35 & 27 & 775.58 & 511.37 \\
\hline CC30 & 316.39 & 20 & 32.51 & 27.64 \\
\hline CC35 & 386.26 & 24 & 133.15 & 104.18 \\
\hline CC40 & 464.28 & 25 & 2934.28 & 942.27 \\
\hline CC45 & 497.68 & 26 & 20641.34 & 3422.21 \\
\hline CC50 & 519.60 & 26 & 45532.40 & 13913.43 \\
\hline CC55 & 581.50 & 28 & 156770.32 & 28429.25 \\
\hline CC60 & 624.95 & 30 & $\geq 2$ weeks & 52801.65 \\
\hline CC65 & 663.31 & 31 & $\geq 2$ weeks & 699831.05 \\
\hline DD30 & 343.65 & 21 & 396.23 & 80.42 \\
\hline DD35 & 410.19 & 22 & 2815.31 & 338.16 \\
\hline DD40 & 490.92 & 25 & 53402.91 & 4618.11 \\
\hline DD45 & 540.17 & 27 & 67293.53 & 7667.97 \\
\hline DD50 & 610.07 & 30 & 186697.41 & 21889.86 \\
\hline DD55 & 639.75 & 29 & $\geq 2$ weeks & 823755.21 \\
\hline
\end{tabular}

computation time increases if the vehicle capacity (i.e., BB compared with $A A$ and $D D$ compared with $C C$ ) or time window width (CC compared with AA and DD compared with $\mathrm{BB}$ ) increases. This is mainly caused as a larger vehicle capacity or time window width will lead to a larger solution space.

In Table 8 we present a more detailed look at the solution of instance AA30. The solution only serves 16 requests in the reported sequence. Since the time dependent travel time instead of the traveled distance is considered as part of the objective, the vehicle's departure time becomes crucial. Therefore, delaying the departure time of the vehicle may lead to less traveling cost. We observe that the vehicle departs from the origin depot at 208.23 and arrives to the destination depot at 581.51. Other departure times will lead to higher travel cost.

In Table 9, we also present the results on small instances if we double the capacity of the vehicles. On one hand, vehicles with more capacity can serve more requests, which returns a better objective than its less capacitate counterparts. On the other hand, for some groups, the instances with larger vehicle capacities need much more time to get the optimal solution (e.g., CC25 and DD25).

\subsection{Performance of the restricted dynamic programing heuristic}

We also have conducted computational experiments to analyze the solution quality produced by the restricted dynamic programing heuristic. In Table 7, we have seen that the exact procedure has enormous computation times. In Table 10, we show the results for the same instances but now with the restricted dynamic programing heuristic. The restricted dynamic programing heuristic algorithm has been run with different values for $B$. In these first tests 
Table 8

Solution of AA30.

\begin{tabular}{lllll}
\hline Start time & End time & Travel cost & Served request & Generated profit \\
\hline 208.23 & 581.51 & 373.28 & $1,11,3,26,22,18,28,25,19,8,5,13,15,7,4,27$ & 640 \\
\hline
\end{tabular}

Table 9

Comparison of the different capacity settings.

\begin{tabular}{|c|c|c|c|c|c|c|c|c|c|c|}
\hline \multirow[b]{2}{*}{ Instances } & \multicolumn{5}{|c|}{ Original Ropke's capacity } & \multicolumn{5}{|c|}{ Double Ropke's capacity } \\
\hline & $\begin{array}{l}\text { Optimal } \\
\text { value }\end{array}$ & Profits & $\begin{array}{l}\text { Requests } \\
\text { served }\end{array}$ & $\begin{array}{l}\text { Travel } \\
\text { cost }\end{array}$ & Time (seconds) & $\begin{array}{l}\text { Optimal } \\
\text { value }\end{array}$ & Profits & $\begin{array}{l}\text { Requests } \\
\text { served }\end{array}$ & Traveling cost & Time (seconds) \\
\hline AA10 & 27.14 & 80 & 2 & 52.86 & 0.23 & 27.14 & 80 & 2 & 52.86 & 0.30 \\
\hline AA15 & 37.77 & 120 & 3 & 82.23 & 0.37 & 79.63 & 200 & 5 & 120.37 & 0.95 \\
\hline AA20 & 73.7 & 240 & 6 & 166.3 & 0.80 & 146.41 & 440 & 11 & 293.59 & 2.57 \\
\hline AA25 & 203.01 & 560 & 14 & 356.99 & 1.59 & 286.68 & 600 & 15 & 313.32 & 29.47 \\
\hline BB10 & 39.26 & 120 & 3 & 80.74 & 0.22 & 46.49 & 200 & 5 & 153.51 & 0.48 \\
\hline BB15 & 99.58 & 280 & 7 & 180.42 & 0.50 & 131.25 & 320 & 8 & 188.75 & 2.65 \\
\hline BB20 & 138.05 & 600 & 15 & 461.95 & 1.95 & 192.46 & 600 & 15 & 407.54 & 11.78 \\
\hline BB25 & 147.88 & 520 & 13 & 372.12 & 2.25 & 268.91 & 800 & 20 & 531.09 & 20.72 \\
\hline $\mathrm{CC} 10$ & 57.63 & 160 & 4 & 102.37 & 0.26 & 96.56 & 240 & 6 & 143.44 & 0.62 \\
\hline CC15 & 98.29 & 280 & 7 & 181.71 & 0.62 & 121.9 & 320 & 8 & 198.1 & 2.74 \\
\hline CC20 & 97.27 & 480 & 12 & 382.73 & 1.25 & 147.50 & 520 & 13 & 372.49 & 5.33 \\
\hline CC25 & 226.23 & 720 & 18 & 493.77 & 11.95 & 434.01 & 960 & 24 & 525.99 & 10808.19 \\
\hline DD10 & 85.88 & 160 & 4 & 74.12 & 0.25 & 99.13 & 160 & 4 & 60.87 & 0.31 \\
\hline DD15 & 99.05 & 200 & 5 & 100.95 & 0.61 & 108.55 & 200 & 5 & 91.45 & 1.37 \\
\hline DD20 & 182.14 & 640 & 16 & 457.86 & 2.11 & 233.61 & 640 & 16 & 406.39 & 19.30 \\
\hline DD25 & 250.16 & 680 & 17 & 429.84 & 199.73 & 452.94 & 960 & 24 & 507.06 & 82620.40 \\
\hline
\end{tabular}

Table 10

Impact of different $B$ values on instances in Table 7 .

\begin{tabular}{|c|c|c|c|c|c|c|c|c|c|c|c|c|c|}
\hline \multirow[b]{2}{*}{ Instance } & \multirow[b]{2}{*}{ Optimal } & \multicolumn{3}{|l|}{$B=1000$} & \multicolumn{3}{|c|}{$B=2500$} & \multicolumn{3}{|c|}{$B=5000$} & \multicolumn{3}{|c|}{$B=10,000$} \\
\hline & & Value & $\begin{array}{l}\text { Time } \\
\text { (seconds) }\end{array}$ & Gap & Value & $\begin{array}{l}\text { Time } \\
\text { (seconds) }\end{array}$ & Gap & Value & $\begin{array}{l}\text { Time } \\
\text { (seconds) }\end{array}$ & Gap & Value & $\begin{array}{l}\text { Time } \\
\text { (seconds) }\end{array}$ & Gap \\
\hline AA30 & 266.72 & 266.72 & 3.32 & 0.00 & 266.72 & 3.03 & 0.00 & 266.72 & 3.14 & 0.00 & 266.72 & 3.01 & 0.00 \\
\hline AA35 & 278.14 & 278.14 & 4.43 & 0.00 & 278.14 & 4.17 & 0.00 & 278.14 & 4.10 & 0.00 & 278.14 & 4.31 & 0.00 \\
\hline AA40 & 341.74 & 341.74 & 4.06 & 0.00 & 341.74 & 5.16 & 0.00 & 341.74 & 5.09 & 0.00 & 341.74 & 4.23 & 0.00 \\
\hline AA45 & 361.58 & 361.58 & 6.65 & 0.00 & 361.58 & 7.40 & 0.00 & 361.58 & 7.68 & 0.00 & 361.58 & 7.89 & 0.00 \\
\hline AA50 & 430.41 & 430.41 & 8.22 & 0.00 & 430.41 & 11.93 & 0.00 & 430.41 & 12.92 & 0.00 & 430.41 & 14.29 & 0.00 \\
\hline AA55 & 436.05 & 433.21 & 11.33 & 0.65 & 436.05 & 15.29 & 0.00 & 436.05 & 18.42 & 0.00 & 436.05 & 23.25 & 0.00 \\
\hline AA60 & 505.02 & 505.02 & 11.19 & 0.00 & 505.02 & 16.68 & 0.00 & 505.02 & 21.76 & 0.00 & 505.02 & 26.66 & 0.00 \\
\hline AA65 & 576.39 & 576.39 & 14.87 & 0.00 & 576.39 & 21.78 & 0.00 & 576.39 & 27.04 & 0.00 & 576.39 & 27.25 & 0.00 \\
\hline AA70 & 606.68 & 606.68 & 15.05 & 0.00 & 606.68 & 25.12 & 0.00 & 606.68 & 30.13 & 0.00 & 606.68 & 33.98 & 0.00 \\
\hline AA75 & 715.53 & 714.65 & 32.07 & 0.12 & 714.65 & 57.36 & 0.12 & 715.53 & 111.54 & 0.00 & 715.53 & 162.37 & 0.00 \\
\hline BB30 & 274.45 & 274.45 & 2.00 & 0.00 & 274.45 & 1.89 & 0.00 & 274.45 & 1.88 & 0.00 & 274.45 & 1.86 & 0.00 \\
\hline BB35 & 337.55 & 337.55 & 1.03 & 0.00 & 337.55 & 1.21 & 0.00 & 337.55 & 1.05 & 0.00 & 337.55 & 1.17 & 0.00 \\
\hline BB40 & 326.82 & 326.82 & 4.01 & 0.00 & 326.82 & 4.79 & 0.00 & 326.82 & 5.07 & 0.00 & 326.82 & 5.37 & 0.00 \\
\hline BB45 & 378.90 & 378.90 & 8.61 & 0.00 & 378.90 & 12.89 & 0.00 & 378.90 & 17.43 & 0.00 & 378.90 & 19.14 & 0.00 \\
\hline BB50 & 432.10 & 432.10 & 8.04 & 0.00 & 432.10 & 14.40 & 0.00 & 432.10 & 19.88 & 0.00 & 432.10 & 22.75 & 0.00 \\
\hline BB55 & 530.84 & 530.84 & 12.40 & 0.00 & 530.84 & 20.19 & 0.00 & 530.84 & 26.80 & 0.00 & 530.84 & 33.32 & 0.00 \\
\hline BB60 & 558.02 & 558.02 & 16.08 & 0.00 & 558.02 & 28.47 & 0.00 & 558.02 & 43.92 & 0.00 & 558.02 & 69.61 & 0.00 \\
\hline BB65 & 547.84 & 547.84 & 16.41 & 0.00 & 547.84 & 31.09 & 0.00 & 547.84 & 61.08 & 0.00 & 547.84 & 104.54 & 0.00 \\
\hline BB70 & 558.29 & 558.29 & 21.72 & 0.00 & 558.29 & 34.10 & 0.00 & 558.29 & 50.89 & 0.00 & 558.29 & 83.74 & 0.00 \\
\hline BB75 & 613.35 & 613.35 & 23.32 & 0.00 & 613.35 & 45.66 & 0.00 & 613.35 & 65.52 & 0.00 & 613.35 & 106.88 & 0.00 \\
\hline СС30 & 316.39 & 316.39 & 9.16 & 0.00 & 316.39 & 13.39 & 0.00 & 316.39 & 16.18 & 0.00 & 316.39 & 18.46 & 0.00 \\
\hline CC35 & 386.26 & 386.26 & 17.39 & 0.00 & 386.26 & 31.84 & 0.00 & 386.26 & 38.27 & 0.00 & 386.26 & 53.09 & 0.00 \\
\hline CC40 & 464.28 & 464.28 & 32.67 & 0.00 & 464.28 & 56.99 & 0.00 & 464.28 & 85.19 & 0.00 & 464.28 & 124.12 & 0.00 \\
\hline CC45 & 497.68 & 497.68 & 52.17 & 0.00 & 497.68 & 108.30 & 0.00 & 497.68 & 190.84 & 0.00 & 497.68 & 303.98 & 0.00 \\
\hline CC50 & 519.60 & 516.59 & 48.71 & 0.58 & 516.59 & 171.45 & 0.58 & 517.10 & 364.55 & 0.48 & 517.10 & 616.42 & 0.48 \\
\hline CC55 & 581.50 & 564.03 & 98.13 & 3.00 & 572.88 & 306.60 & 1.48 & 572.88 & 746.83 & 1.48 & 581.50 & 1416.82 & 0.00 \\
\hline CC60 & 624.95 & 593.46 & 114.55 & 5.04 & 606.11 & 327.94 & 3.01 & 624.95 & 911.43 & 0.00 & 624.95 & 1840.42 & 0.00 \\
\hline CC65 & 663.31 & 636.12 & 169.39 & 4.10 & 653.51 & 470.64 & 1.48 & 655.84 & 1295.07 & 1.13 & 662.61 & 3879.84 & 0.11 \\
\hline CC70 & - & 678.11 & 188.24 & $\geq 0.55$ & 680.30 & 585.96 & $\geq 0.23$ & 680.56 & 1589.12 & $\geq 0.19$ & 681.84 & 4858.95 & $\geq 0.00$ \\
\hline CC75 & - & 686.68 & 250.89 & $\geq 0.00$ & 686.68 & 634.38 & $\geq 0.00$ & 686.68 & 1745.03 & $\geq 0.00$ & 686.68 & 7159.92 & $\geq 0.00$ \\
\hline DD30 & 343.65 & 343.65 & 17.32 & 0.00 & 343.65 & 35.09 & 0.00 & 343.65 & 46.71 & 0.00 & 343.65 & 46.1 & 0.00 \\
\hline DD35 & 410.19 & 410.19 & 37.57 & 0.00 & 405.07 & 92.01 & 1.25 & 410.19 & 168.55 & 0.00 & 410.19 & 275.19 & 0.00 \\
\hline DD40 & 490.92 & 477.82 & 74.59 & 2.67 & 490.92 & 233.52 & 0.00 & 490.92 & 526.53 & 0.00 & 490.92 & 1026.12 & 0.00 \\
\hline DD45 & 540.17 & 540.17 & 78.77 & 0.00 & 540.17 & 210.87 & 0.00 & 540.17 & 415.69 & 0.00 & 540.17 & 953.24 & 0.00 \\
\hline DD50 & 610.07 & 589.67 & 104.49 & 3.34 & 599.02 & 264.49 & 1.81 & 610.07 & 725.41 & 0.00 & 610.07 & 1277.29 & 0.00 \\
\hline DD55 & 639.75 & 617.52 & 159.5 & 3.47 & 626.86 & 550.42 & 2.01 & 629.70 & 1132.39 & 1.57 & 639.75 & 2754.41 & 0.00 \\
\hline DD60 & - & 690.87 & 191.22 & $\geq 3.16$ & 695.74 & 742.63 & $\geq 2.47$ & 695.54 & 1785.51 & $\geq 2.50$ & 713.39 & 4054.79 & $\geq 0.00$ \\
\hline DD65 & - & 754.65 & 246.71 & $\geq 0.75$ & 758.57 & 963.77 & $\geq 0.23$ & 760.32 & 2212.53 & $\geq 0.00$ & 760.32 & 5853.15 & $\geq 0.00$ \\
\hline DD70 & - & 746.44 & 334.40 & $\geq 2.31$ & 751.56 & 1434.89 & $\geq 1.64$ & 753.58 & 3869.98 & $\geq 1.38$ & 764.10 & 9581.94 & $\geq 0.00$ \\
\hline DD75 & - & 830.50 & 399.73 & $\geq 0.63$ & 831.49 & 1899.06 & $\geq 0.51$ & 835.76 & 4816.39 & $\geq 0.00$ & 835.76 & 15842.28 & $\geq 0.00$ \\
\hline Average & 515.18 & 510.34 & 71.26 & $\geq 0.76$ & 512.48 & 237.42 & $\geq 0.42$ & 513.72 & 580.44 & $\geq 0.22$ & 515.10 & 1567.30 & $\geq 0.01$ \\
\hline
\end{tabular}


Table 11

Impact of different $E$ values on instances when $B=10,000$.

\begin{tabular}{|c|c|c|c|c|c|c|c|c|c|}
\hline \multirow[b]{2}{*}{ Instance } & \multicolumn{3}{|l|}{$E=0.5 n$} & \multicolumn{3}{|c|}{$E=0.25 n$} & \multicolumn{3}{|c|}{$E=0.125 n$} \\
\hline & Value & Time (seconds) & Gap & Value & Time (seconds) & Gap & Value & Time (seconds) & Gap \\
\hline AA30 & 266.72 & 4.23 & 0.00 & 258.68 & 3.99 & 3.01 & 183.89 & 1.33 & 31.06 \\
\hline AA35 & 278.14 & 5.01 & 0.00 & 278.14 & 4.73 & 0.00 & 268.40 & 2.14 & 3.50 \\
\hline AA40 & 341.74 & 7.27 & 0.00 & 341.74 & 6.93 & 0.00 & 341.74 & 4.57 & 0.00 \\
\hline AA45 & 361.58 & 8.88 & 0.00 & 361.58 & 8.49 & 0.00 & 358.74 & 8.22 & 0.79 \\
\hline AA50 & 430.41 & 20.11 & 0.00 & 430.41 & 11.01 & 0.00 & 418.09 & 9.19 & 2.86 \\
\hline AA55 & 436.05 & 33.49 & 0.00 & 436.05 & 26.04 & 0.00 & 424.6 & 13.15 & 2.63 \\
\hline AA60 & 505.02 & 31.72 & 0.00 & 505.02 & 23.89 & 0.00 & 505.02 & 14.34 & 0.00 \\
\hline AA65 & 576.39 & 30.23 & 0.00 & 576.39 & 24.45 & 0.00 & 568.4 & 22.18 & 1.39 \\
\hline AA70 & 606.68 & 79.56 & 0.00 & 606.68 & 47.77 & 0.00 & 599.97 & 31.50 & 1.11 \\
\hline AA75 & 715.53 & 294.76 & 0.00 & 715.53 & 210.50 & 0.00 & 713.67 & 144.16 & 0.26 \\
\hline BB30 & 274.45 & 3.53 & 0.00 & 274.45 & 2.20 & 0.00 & 249.00 & 1.48 & 9.27 \\
\hline BB35 & 337.55 & 2.62 & 0.00 & 316.85 & 5.02 & 6.13 & 316.85 & 1.89 & 6.13 \\
\hline BB40 & 326.82 & 6.6 & 0.00 & 326.82 & 6.52 & 0.00 & 326.82 & 4.54 & 0.00 \\
\hline BB45 & 378.90 & 25 & 0.00 & 378.9 & 14.61 & 0.00 & 378.9 & 11.62 & 0.00 \\
\hline BB50 & 432.10 & 32.89 & 0.00 & 432.10 & 17.19 & 0.00 & 432.1 & 11.83 & 0.00 \\
\hline BB55 & 530.84 & 50.25 & 0.00 & 530.84 & 31.97 & 0.00 & 530.84 & 18.36 & 0.00 \\
\hline BB60 & 558.02 & 72.85 & 0.00 & 558.02 & 71.65 & 0.00 & 558.02 & 36.90 & 0.00 \\
\hline BB65 & 547.84 & 129.72 & 0.00 & 547.84 & 120.02 & 0.00 & 547.84 & 56.83 & 0.00 \\
\hline BB70 & 558.29 & 139.86 & 0.00 & 558.29 & 92.68 & 0.00 & 558.29 & 48.46 & 0.00 \\
\hline BB75 & 613.35 & 190.45 & 0.00 & 613.35 & 158.15 & 0.00 & 613.35 & 49.19 & 0.00 \\
\hline CC30 & 316.39 & 20.66 & 0.00 & 316.39 & 19.25 & 0.00 & 292.65 & 5.80 & 7.50 \\
\hline CC35 & 386.26 & 54.93 & 0.00 & 386.26 & 45.49 & 0.00 & 375.17 & 12.00 & 2.87 \\
\hline CC40 & 464.28 & 133.99 & 0.00 & 464.28 & 152.86 & 0.00 & 448 & 38.50 & 3.51 \\
\hline CC45 & 497.68 & 497.68 & 0.00 & 497.68 & 352.53 & 0.00 & 479.19 & 191.45 & 3.72 \\
\hline CC50 & 517.1 & 542.93 & 0.48 & 517.1 & 598.14 & 0.48 & 503.47 & 421.23 & 3.10 \\
\hline CC55 & 581.5 & 1920.55 & 0.00 & 581.5 & 1421.72 & 0.00 & 579.34 & 2338.11 & 0.37 \\
\hline CC60 & 624.95 & 1244.56 & 0.00 & 624.95 & 1579.68 & 0.00 & 624.95 & 2338.36 & 0.00 \\
\hline CC65 & 662.61 & 4098.04 & 0.11 & 662.61 & 2629.06 & 0.11 & 662.61 & 2203.57 & 0.11 \\
\hline CC70 & 681.84 & 4918.55 & 0.00 & 681.84 & 3110.90 & 0.00 & 681.84 & 2453.23 & 0.00 \\
\hline CC75 & 686.68 & 4290.45 & 0.00 & 686.68 & 5518.81 & 0.00 & 686.68 & 3183.47 & 0.00 \\
\hline DD30 & 343.65 & 68.19 & 0.00 & 343.65 & 63.74 & 0.00 & 330.79 & 17.25 & 3.74 \\
\hline DD35 & 410.19 & 350.53 & 0.00 & 410.19 & 399.22 & 0.00 & 385.85 & 84.60 & 5.93 \\
\hline DD40 & 490.92 & 1158.38 & 0.00 & 490.92 & 1133.28 & 0.00 & 483.48 & 1036.05 & 1.52 \\
\hline DD45 & 540.17 & 889.37 & 0.00 & 540.17 & 962.77 & 0.00 & 532.17 & 827.67 & 1.48 \\
\hline DD50 & 610.07 & 1919.31 & 0.00 & 610.07 & 1595.29 & 0.00 & 597.37 & 1352.25 & 2.08 \\
\hline DD55 & 639.75 & 2861.15 & 0.00 & 639.75 & 2733.71 & 0.00 & 638.36 & 4879.35 & 0.22 \\
\hline DD60 & 713.39 & 5432.74 & 0.00 & 713.39 & 4656.094 & 0.00 & 708.52 & 4264.71 & 0.68 \\
\hline DD65 & 760.32 & 6456.7 & 0.00 & 760.32 & 5255.72 & 0.00 & 758.57 & 4545.88 & 0.23 \\
\hline DD70 & 764.1 & 9593.25 & 0.00 & 764.1 & 8355.49 & 0.00 & 760.5 & 6054.51 & 0.47 \\
\hline DD75 & 835.76 & 11762.83 & 0.00 & 835.76 & 10770.09 & 0.00 & 835.76 & 8465.89 & 0.00 \\
\hline Average & 515.10 & 1484.60 & 0.01 & 514.38 & 1306.04 & 0.15 & 506.50 & 1130.14 & 1.69 \\
\hline
\end{tabular}

we set $E=\infty$. For the instances of which the optimal solution is not known, we compare the solution to the best found solution (i.e., the solution found in case $B=10,000$ ) to have a lower bound on the gap.

With a value of $B=1000$ the algorithm leads in on average 71.26 seconds to the optimal solution for 25 out of the 40 instances. For 1 instance it is not sure whether or not the optimal solution is reached. The average gap is at least $0.79 \%$. With a value of $B=2500$ the average computation time increased to $237.42 \mathrm{sec}-$ onds. For one more instance the optimal solution is found, and again there is 1 instance for which it is not sure whether or not the optimal solution is reached. The average gap (at least $0.42 \%$ ) is about half of the gap as in the case of $B=1000$. With a value of $B=5000$ the average gap is halved again to at least $0.22 \%$ and the number of instances for which the optimal solution is found increases considerably to 30 . For 3 more instances the solution found could be the optimal one. However, the average computation time is also increased to 580.44 seconds. Increasing the value of $B$ to 10,000 ensures that 32 of the 34 instances of which we know the optimal solution are solved to optimality. The average computation time in case $B=10,000$ equals 1567.3 seconds. The two instances which were not solved to optimality have, respectively, a gap of $0.48 \%$ and $0.11 \%$. This means that, compared to the tailored labeling algorithm, the average gap of the restricted dynamic programing heuristic algorithm over the 34 instances of which the optimal solution is known is just $0.02 \%$.

Note that all the instances for which the exact labeling algorithm was not able to solve in 2 weeks (e.g., DD75 in Table 7), can be solved within 20 hours with this restricted dynamic programing algorithm.

In Table 11, we present the impact of different $E$ values on the performances of the algorithm with $B=10,000$. We set the values of $E$ to $0.5 n, 0.25 n$ and $0.125 n$ and fractional values are rounded to its closed integer.

The results indicate that the computation times decrease if the value for $E$ decreases, but not that much. As can be seen in Table 11 , when the value of $E$ is equal to $0.5 n$, the solution quality is the same as in case $E=\infty$ but the computation times are reduced by $5 \%$. When we restrict $E$ further the solution quality is going down drastically without gaining too much computation time. In comparison, a combination of $B=1000$ and $E=\infty$ is much faster and has higher solution quality then the combination of $B=10,000$ and $E=0.125 n$

\section{Conclusion}

This paper presents the time-dependent capacitated profitable tour problem with time windows and precedence constraints, which combines features of both the traveling salesman problem 
with pickup and delivery, and the traveling salesman problem with profits. Moreover, time-dependent traveling speeds are considered to capture road congestion, which increases the complexity of the problem.

We propose a tailored labeling algorithm to solve this problem enriched by new and strong dominance rules to discard $95 \%$ of the labels. Extensive computational results show that most instances with up to 75 requests can be solved to optimality within the given time limit of two weeks, but also show that some instances remain unsolved.

To reduce the computation time and memory usage, a restricted dynamic programing heuristic algorithm is implemented. The heuristic is able to find solutions for all instances with good qualities (on average 0.01\% gap) and less computational time (on average 1567 seconds).

Obviously, the performance of our exact algorithm critically depends on the tailored dominance criterion that we propose. It shows great potential when there are relatively tight time windows attached to all the nodes. Further strengthening dominance is a promising direction for future research.

In addition, solving extensions of the time-dependent capacitated profitable tour problem with time windows and precedence constraints seems to be an attractive research direction. More specifically, the time-dependent variant of the pickup and delivery problem with time windows (TDPDPTW) and the time-dependent team orienteering problem (TDTOP) are very interesting as it aims to optimize the routes of a fleet of vehicles, instead of a single vehicle only. When column generation is utilized to solve those two problems in an exact way, our proposed model can be used as the pricing problem.

\section{Acknowledgments}

The authors gratefully acknowledge funds provided by China Scholarship Council (CSC) under Grant No. 201206160092.

\section{References}

Balas, E. (1989). The prize-collecting traveling salesman problem. Networks, 19(6), 621-636.

Bisiani, R. (1987). Beam search. In S. Shapiro (Ed.), Encyclopedia of artificial intelligence (pp. 56-58). Wiley and Sons.

Dabia, S., Ropke, S., van Woensel, T., \& de Kok, T. (2013). Branch and cut and price for the time dependent vehicle routing problem with time windows. Transportation Science, 47(3), 380-396.

Dell' Amico, M., Maffioli, F., \& Varbrand, P. (1995). On prize-collecting tours and the asymmetric traveling salesman problem. International Transactions in Operational Research, 2(3), 297-308.

Donati, A., Montemanni, R., Casagrande, N., Rizzolo, A. E., \& Gambardella, L. (2008). Time dependent vehicle routing problem with a muti-ant colony system. European Journal of Operational Research, 185(3), 1174-1191.
Dumitrescu, I., Ropke, S., \& Cordeau, J. (2010). The traveling salesman problem with pickup and delivery: polyhedral results and branch-and-cut algorithm. Mathematic Programming Series A, 121(2), 269-305.

Feillet, D., Dejax, P., \& Gendreau, M. (2004). An exact algorithm for the elementary shortest path problem with resource constraints: Application to some vehicle routing problems. Networks, 44(3), 216-229.

Feillet, D., Dejax, P., \& Gendreau, M. (2005). Traveling salesman problems with profits. Transportation Science, 39(2), 188-205.

Fomin, F., \& Lingas, A. (2002). Approximation algorithms for time-dependent orienteering. Information Processing Letters, 83(2), 57-62.

Gromicho, J., van Hoorn, J., Kok, A., \& Schutten, J. (2012). Restricted dynamic programming: A flexible framework for solving realistic VRPs. Computers and Operations Research, 39(5), 902-909.

Gunawan, A., Lau, H. C., \& Vansteenwegen, P. (2016). Orienteering problem: A survey of recent variants, solution approaches and applications. European Journal of Operational Research, 255(2), 315-332.

baraki, T. Imahori, S., Nonobe, K., Sobue, K, Uno, T, \& Yagiura, M. (2008). An iterated local search algorithm for the vehicle routing problem with convex time penalty functions. Discrete Applied Mathematics, 156(11), 2050-2069.

Ichoua, S., Gendreau, M., \& Potvin, J. (2003). Vehicle dispatching with time-dependent travel times. European Journal of Operational Research, 144(2), 379-396.

Laporte, G., \& Martello, S. (1990). The selective traveling salesman problem. Discrete Applied Mathematics, 26(2-3), 193-207.

Li, J. (2011). Model and algorithm for time-dependent team orienteering problem. In S. Lin, \& X. Huang (Eds.), Advanced research on computer education, simulation and modeling. Communications in computer and information science: vol. 175 (pp. 1-7).

Malandraki, C., \& Daskin, M. (1992). Time dependent vehicle routing problems: Formulations, properties, and heuristic algorithms. Transportation Science, 26(3), 185-200.

Malandraki, C., \& Dial, R. (1996). A restricted dynamic programming heuristic algorithm for the time dependent traveling salesman problem. European Journal of Operational Research, 90(1), 45-55.

Righini, G., \& Salani, M. (2006). Symmetry helps: Bounded bi-directional dynamic programming for the elementary shortest path problem with resource constraints. Discrete Optimization, 3(3), 255-273.

Ropke, S., Cordeau, J.-F., \& Laporte, G. (2009). Branch and cut and price for the pickup and delivery problem with time windows. Transportation Science, 43(3), 267-286.

Ruland, K., \& Rodin, E. (1997). The pickup and delivery problem: Faces and branch-and-cut algorithm. Computers Mathematics with Applications, 33(12), $1-13$.

Ruland, K. S. (1994). Polyhedral solution to the pickup and delivery problem. Ph.D. thesis, Sever Institute, Washington University in St.Louis.

Sigurd, M., \& Pisinger, D. (2004). Scheduling transportation of live animals to avoid the spread of diseases. Transportation Science, 38(2), 197-209.

Sol, M. (1994). Column generation techniques for pickup and delivery problems. Ph.D thesis, Technische Universiteit Eindhoven.

Taș, D., Dellaert, N., van Woensel, T., \& de Kok, T. (2014). The time-dependent vehicle routing problem with soft time windows and stochastic travel times. Transportation Research Part C: Emerging Technologies, 48, 66-83.

Vansteenwegen, P., Souffriau, W., \& Oudheusden, D. V. (2011). The orienteering problem: a survey. European Journal of Operational Reseach, 209(1), 1-10.

Verbeeck, C., Aghezzaf, E. H., \& Vansteenwegen, P. (2014). A fast solution method for the time-dependent orienteering problem with time windows. European Journal of Operational Research, 236(2), 419-432.

Verbeeck, C., Vansteenwegen, P., \& Aghezzaf, E.-H. (2016). Solving the stochastic time-dependent orienteering problem with time windows. European Journal of Operational Research, 255(3), 699-718.

van Woensel, T., Kerbache, L., Peremans, H., \& Vandaele, N. (2008). Vehicle routing with dynamic travel times: A queueing approach. European Journal of Operational Research, 186(3), 990-1007. 